\title{
Ribosome profiling in mouse hippocampus: plasticity-induced regulation and bidirectional control by TSC2 and FMRP
}

\author{
Annie Hien ${ }^{1,2+}\left(\mathbb{D}\right.$, Gemma Molinaro $^{3+} \mathbb{D}$, Botao Liu ${ }^{1}$, Kimberly M. Huber ${ }^{3^{*}}\left(\mathbb{D}\right.$ and Joel D. Richter ${ }^{1 *}(\mathbb{D})$
}

\begin{abstract}
Background: Mutations in TSC2 are the most common cause of tuberous sclerosis (TSC), a disorder with a high incidence of autism and intellectual disability. TSC2 regulates mRNA translation required for group 1 metabotropic glutamate receptor-dependent synaptic long-term depression (mGluR-LTD) and behavior, but the identity of mRNAs responsive to mGluR-LTD signaling is largely unknown.

Methods: We utilized TSC2 ${ }^{+/}$mice as a mouse model of TSC and prepared hippocampal slices from these animals. We induced mGluR-LTD synaptic plasticity in slices and processed the samples for RNA-seq and ribosome profiling to identify differentially expressed genes in $\mathrm{TsC2}^{+/-}$and following mGluR-LTD synaptic plasticity.

Results: Ribosome profiling reveals that in $\mathrm{TsC}^{+/-}$mouse hippocampal slices, the expression of several mRNAs was dysregulated: terminal oligopyrimidine (TOP)-containing mRNAs decreased, while FMRP-binding targets increased. Remarkably, we observed the opposite changes of FMRP binding targets in Fmr1-ly hippocampi. In wild-type hippocampus, induction of mGluR-LTD caused rapid changes in the steady-state levels of hundreds of mRNAs, many of which are FMRP targets. Moreover, mGluR-LTD failed to promote phosphorylation of eukaryotic elongation factor 2 (eEF2) in TSC mice, and chemically mimicking phospho-eEF2 with low cycloheximide enhances mGluR-LTD in TSC mice.

Conclusion: These results suggest a molecular basis for bidirectional regulation of synaptic plasticity and behavior by TSC2 and FMRP. Our study also suggests that altered mGluR-regulated translation elongation contributes to impaired synaptic plasticity in $\mathrm{SSC2}^{+/-}$mice.
\end{abstract}

\section{Background}

Tuberous sclerosis (TSC) is an autosomal dominant disorder characterized by benign tumor growth in multiple organs and neuropsychiatric symptoms. Individuals with TSC have an increased incidence of seizures $(\sim 90 \%)$,

\footnotetext{
*Correspondence: Kimberly.Huber@UTSouthwestern.edu; Joel. Richter@umassmed.edu

${ }^{\dagger}$ Annie Hien and Gemma Molinaro contributed equally to this work

${ }^{1}$ Program in Molecular Medicine, University of Massachusetts Medical School, Worcester, MA 01605, USA

${ }^{3}$ Department of Neuroscience, University of Texas Southwestern Medical Center, Dallas, TX 75390, USA

Full list of author information is available at the end of the article
}

intellectual disability $(\sim 50 \%)$, and autism $(\sim 50 \%)$ [1]. Disrupted neuronal circuitry likely underlies many neuropathologies in TSC because individuals with an anatomically normal brain can still present substantial developmental delay, intellectual disability, and autism. More direct evidence for impaired neuronal connectivity is derived from mouse models of the disorder, which display hyperexcitability, aberrant synaptic plasticity, and altered dendritic spine morphology [2,3]. TSC is caused by loss-of-function mutations in TSC1 or TSC2; mutations in TSC2 are more common and are responsible for the most severe symptoms. The TSC1/2 proteins heterodimerize to form the tuberous sclerosis complex (TSC), original author(s) and the source, provide a link to the Creative Commons licence, and indicate if changes were made. The images or other third party material in this article are included in the article's Creative Commons licence, unless indicated otherwise in a credit line to the material. If material is not included in the article's Creative Commons licence and your intended use is not permitted by statutory regulation or exceeds the permitted use, you will need to obtain permission directly from the copyright holder. To view a copy of this licence, visit http://creativecommons.org/licenses/by/4.0/. The Creative Commons Public Domain Dedication waiver (http://creativeco mmons.org/publicdomain/zero/1.0/) applies to the data made available in this article, unless otherwise stated in a credit line to the data. 
a GTPase-activating protein that inhibits Ras homology enriched in brain (Rheb) $[4,5]$. Rheb activates the mechanistic target of rapamycin (mTOR), a kinase that forms two biochemically distinct complexes: mTORC1 and mTORC2 [6]. mTORC1 regulates mRNA translation primarily via activation of the kinase p70S6K to phosphorylate rpS6 and 4E-BP [7-9]. The less-characterized mTORC2 plays a role in actin cytoskeletal reorganization [6]. Complete loss of TSC1 or TSC2 leads to excessive mTOR activity, and mutations in other components of the mTOR pathway are also linked to autism in humans such as PTEN, RHEB, and MTOR $[10,11]$. Thus, the mTOR pathway forms a highly connected signaling network linked to autism. Although pharmacological options are available for the treatment for seizures in individuals with TSC, there currently is no effective pharmacological treatment for the neuropsychiatric symptoms of intellectual disability and autism.

The mTOR pathway integrates signaling from various inputs to regulate synaptic plasticity and higher cognitive function. One example is metabotropic glutamate receptor-mediated long-term depression (mGluR-LTD), a form of synaptic plasticity that requires de novo protein synthesis [12]. The mGluR1/5 agonist dihydroxyphenylglycine (DHPG) stimulates group 1 metabotropic glutamate receptors to activate the PI3K/AKT/mTOR pathway, leading to translation of largely unidentified mRNAs required for mGluR-LTD [13, 14]. Multiple mouse models of autism, such as TSC and Fragile X Syndrome, have altered hippocampal mGluR-dependent synaptic plasticity [2, 15-17]. Although experiments with rapamycin and inhibitors of protein synthesis suggest that excessive mTORC1-dependent translation drives the altered synaptic plasticity phenotype in TSC mice, other findings suggest a more complex picture. For example, $T s c 2^{+/-}$mice have reduced hippocampal protein synthesis rates instead of the predicted excessive protein synthesis from mTOR hyperactivation [2]. In addition, a recent study challenged the role of mTORC1 in hippocampal mGluR-LTD, arguing that mTORC2 is the major regulator of this form of synaptic plasticity [18, 19] but see [13, 20]. MGluR1/5 signaling can also regulate translation factors to suppress global protein synthesis, such as phosphorylation of eukaryotic initiation factor 2 alpha and eukaryotic elongation factor 2 (eEF2), which are both necessary for mGluR-LTD [18, 21]. Although mGluR1/5 stimulation is known to promote translation of specific mRNAs [22], how translation is regulated in a transcriptome-wide manner by mGluR $1 / 5$ in either the normal or TSC brain is unknown.

To study the role of TSC2 in synaptic plasticity, we generated a germline $T s c 2^{+-}$mouse and performed ribosome profiling, an unbiased whole transcriptome method that determines the number and positions of ribosomes on all mRNAs and thus serves as a proxy for protein synthesis [23]. Together with RNA-seq, ribosome profiling in wild-type and $T s c 2^{+/-}$hippocampal slices under basal conditions and after induction of mGluR-LTD allows us to define posttranscriptional changes in gene expression in response to synapse activation [12]. We chose the $T s c 2^{+/-}$mouse model, because we strove to capture translational dysregulation that arises from TSC2 haploinsufficiency as in humans with TSC. This mouse model displays excessive mTOR signaling, a well-characterized deficient mGluR synaptic plasticity, and alterations in social interactions and hippocampal-dependent learning tasks that are reversed by rapamycin treatment $[2$, 24-26]. Using ribosome profiling, we identified changes in the $T s c 2^{+/-}$hippocampus as well as following mGluRLTD signaling. We also observed that mGluR1/5 stimulation induces a rapid increase in mRNA levels, and targets of Fragile X Mental Retardation Protein (FMRP), an RNA-binding protein whose loss causes Fragile X Syndrome, are enriched in this group. mGluR stimulation also promotes phosphorylation of eukaryotic elongation factor (eEF2), which is deficient in $T s c 2^{+/-}$mice. These animals have a reduced mGluR-LTD, and chemically mimicking phospho-eEF2 enhances this form of synaptic plasticity. Our results suggest a novel pathway of translational dysregulation in ribosome translocation (polypeptide elongation) that may contribute to altered translation and synaptic plasticity in TSC mice.

\section{Methods \\ Animals}

Germline $T s c 2^{+/-}$mice were generated by crossing CMV-Cre (B6.C-Tg(CMV-cre)1Cgn/J) (JAX stock number: 006054) with Tsc2flox mice (JAX Stock number: 027458). F1 was then bred on the C57BL/6 J background to obtain WT and $T s c 2^{+/-}$mice. Animals were given ad libitum access to food and water and reared on a 12-h light-dark cycle. All experiments were approved by the Institutional Animal Care and Use Committee at University of Texas Southwestern and conducted in accordance with the National Institutes of Health Principles of Laboratory Animal Care.

\section{Hippocampal slice preparation}

Acute hippocampal brain slices were prepared from P3040 wild-type (WT) and $T s c 2^{+/-}$littermates as described previously [27]. Briefly, mice were anesthetized with ketamine $(125 \mathrm{mg} / \mathrm{kg}) / \mathrm{xylazine}(25 \mathrm{mg} / \mathrm{kg})$ and transcardially perfused with chilled $\left(4{ }^{\circ} \mathrm{C}\right)$ sucrose dissection buffer containing the following (in $\mathrm{mm}$ ): $2.6 \mathrm{KCl}, 1.25$ $\mathrm{NaH}_{2} \mathrm{PO}_{4}, 26 \mathrm{NaHCO}_{3}, 0.5 \mathrm{CaCl}_{2}, 5 \mathrm{MgCl}_{2}, 212$ sucrose, and 10 dextrose aerated with $95 \% \mathrm{O}_{2} / 5 \% \mathrm{CO}_{2}$. Transverse 
hippocampal slices $(400 \mu \mathrm{m})$ were obtained on a Leica VT1200S slicer. CA3 was cut off to avoid epileptogenic activity induced by DHPG. Slices were recovered for 3-4 $\mathrm{h}$ and maintained at $30^{\circ} \mathrm{C}$ in artificial cerebral spinal fluid (ACSF) containing the following (in $\mathrm{mM}$ ): $119 \mathrm{NaCl}$, $2.5 \mathrm{KCl}, 2 \mathrm{CaCl}_{2}, 1 \mathrm{MgCl}_{2}, 26 \mathrm{NaHCO}_{3}, 1 \mathrm{NaH}_{2} \mathrm{PO}_{4}$, and 11 D-glucose aerated with $95 \% \mathrm{O}_{2} / 5 \% \mathrm{CO}_{2}$ to $\mathrm{pH}$ 7.4. Slices were then treated with cycloheximide $(100 \mu \mathrm{g} /$ $\mathrm{ml}$ ) in ACSF, $4{ }^{\circ} \mathrm{C}$, snap-frozen on dry ice/EtOH bath, and stored at $-80{ }^{\circ} \mathrm{C}$. Alternatively, hippocampal slices were treated with DHPG $(100 \mu \mathrm{M})$ (Tocris) or artificial cerebrospinal fluid (ACSF, vehicle) for $5 \mathrm{~min}$. For the cycloheximide experiments, hippocampal slices were pretreated with $75 \mathrm{nM}$ cycloheximide for $45-60 \mathrm{~min}$. At the end of the incubation, CA1 was dissected out, snapfrozen in a dry ice/ethanol bath and stored at $-80{ }^{\circ} \mathrm{C}$. Some hippocampal slices were prepared without removing CA3 and used for RNA-seq and ribosome profiling experiments as indicated below (batches 1-5).

\section{Electrophysiology recordings}

For all recordings, slices were submerged and perfused with ACSF at $2.5-3.5 \mathrm{ml} / \mathrm{min}\left(30 \pm 1{ }^{\circ} \mathrm{C}\right)$. Field potentials (FPs) and EPSCs were evoked by stimulation of the Schaffer collateral pathway with a concentric bipolar tungsten electrode. Field potentials were recorded with a glass electrode $(1 \mathrm{M} \Omega$ ) filled with ACSF placed in the stratum radiatum of CA1. Test stimuli were delivered every $30 \mathrm{~s}$, and a stable baseline was obtained at $\sim 50 \%$ of the maximum FP amplitude.

\section{Western Blotting}

Western blotting was performed on whole hippocampus homogenates or on CA1 hippocampal slices, where indicated, and as previously described [14]. CA1 hippocampal slices were lysed with lysis buffer $(50 \mathrm{~mm}$ Tris, $\mathrm{pH}$ 7.4, $120 \mathrm{~mm} \mathrm{NaCl}, 50 \mathrm{~mm} \mathrm{NaF}$, and 1\% Triton X-100, containing Protease Inhibitor Mixture, Sigma \#P8340, and phosphatase inhibitor mixture 2 and 3, Sigma \#P5726 and \#P0040). Samples were homogenized using brief (5-10 s) pulses of sonication with an ultrasonic cell disruptor until lysates were clear. Lysates were then centrifuged at $15,700 \times g$ for $10 \mathrm{~min}$ at $4{ }^{\circ} \mathrm{C}$, the pellets were discarded, and protein levels in the supernatant were measured using a Pierce BCA kit. After SDS-PAGE, proteins were transferred onto PVDF membranes. After blocking with $5 \%$ BSA in $1 \times$ TBS, $0.05 \%$ Tween 20 for $1 \mathrm{~h}$, membranes were incubated with the following primary antibodies in blocking buffer overnight at $4{ }^{\circ} \mathrm{C}$ : The following antibodies were used in this study: S6 Ribosomal Protein (Cell Signaling, \#2217), Phospho-S6 Ribosomal Protein Ser235/236 (Cell Signaling, \#4856), Akt (Cell Signaling, \#9272), Phospho-Akt Ser473 (Cell Signaling,
\#4060), p44/42 MAPK (Erk1/2) (Cell Signaling \#9102), Phospho-p44/42 MAPK (Erk1/2) Thr202/Tyr204 (Cell Signaling, \#9101), eEF2 (Cell Signaling, \#2332), PhosphoeEF2 Thr56 (Cell Signaling, \#2331), mTOR (Cell Signaling, \#2983), Phospho-mTOR Ser2448 (Cell Signaling, \#2971), 4E-BP1 (Cell Signaling, \#9452), Phospho-4E-BP1 Thr37/46 (Cell Signaling, \#9459), RPL4 (Proteintech, \#11302-1-AP), RPS25 (Sigma, \#HPA031801-100UL), LARP1 (Proteintech, \#13708-1-AP), FMRP (DSHB, 2F51), Glyceraldehyde-3-Phosphate Dehydrogenase (Millipore, \#MAB374), actin (Millipore, \#MAB1501). After three $10 \mathrm{~min}$ washes of $1 \times \mathrm{TBS}, 0.05 \%$ Tween 20 , membranes were incubated with the appropriate HRP-conjugated secondary antibodies in $5 \%$ milk in $1 \times$ TBS, $0.05 \%$ Tween 20 for $1 \mathrm{~h}$ at room temperature. After three $10 \mathrm{~min}$ washes of $1 \times \mathrm{TBS}, 0.05 \%$ Tween 20 , the membranes were developed using ECL. For comparison of phosphorylated protein levels across conditions or genotypes, immunoreactive phosphorylated protein bands were normalized to total protein levels from the same slice homogenates (e.g., P-ERK/ERK), each of which was first normalized to loading control (either GAPDH or actin where indicated).

\section{Sample preparation for RiboSeq and RNA-Seq}

Hippocampal slices from six mice were pooled, lysed in polysome buffer (as above but with the addition of $100 \mathrm{ug} / \mathrm{mL}$ cycloheximide, $25 \mathrm{U} / \mathrm{mL}$ Turbo Dnase I, $1 \times$ EDTA-free protease inhibitor, $1 \%$ NP-40), and triturated with a $25 \mathrm{G}$ needle ten times. Following placement on ice for $10 \mathrm{~min}$, the lysate was centrifuged $2000 \mathrm{~g}$ for $10 \mathrm{~min}$ at $4{ }^{\circ} \mathrm{C}$ and the supernatant collected and centrifuged again for a $20,000 \mathrm{~g}$ for $10 \mathrm{~min}$ at $4{ }^{\circ} \mathrm{C}$. This supernatant was collected and the RNA concentration measured using the Qubit RNA HS Assay kit (Invitrogen, \#Q32852). One-fourth of the lysate was saved for RNA-seq libraries ( $\sim 1$ ug of RNA), and the remainder $(\sim 3.5 \mathrm{ug})$ was treated with 11.3 ug of RNase A (Ambion, \#AM2270) and 1410U of Rnase T1 (Thermo Fisher Scientific, \#EN0542), which corresponds to a ratio of $\sim$ 3ug Rnase A/375U Rnase T1 per ug of input RNA. The Rnase treatment was carried out at $25^{\circ} \mathrm{C}$ for $30 \mathrm{~min}$ with gentle shaking and the digest stopped by placement on ice and the addition of $2.5 \mathrm{u} \mathrm{L}$ of SuperRNaseIn (Invitrogen, \#AM2696). The lysate was layered over a linear $10-50 \%$ sucrose gradient (prepared with polysome buffer supplemented with $1 \mathrm{mM}$ DTT and $100 \mathrm{ug} / \mathrm{mL} \mathrm{CHX}$ ) and centrifuged at 35,000 rpm for $2.5 \mathrm{~h}$ at $4{ }^{\circ} \mathrm{C}$ in a SW41 rotor. The $80 \mathrm{~S}$ monosome fractions were identified by continuous A260 monitoring and pooled for RNA extraction by TRIzol LS. To aid in RNA recovery of low concentration samples, 20 ug glycogen was added to all precipitation steps. A total of three biological replicates per genotype and treatment were made from hippocampal slices (CA1 enriched, batch 6-8) from 
$T s c 2^{+/-}$and their wild-type littermate mice. Wild-type slices had a total of eight biological replicates for RiboSeq and 7 for RNA-Seq (batch 1-5 from hippocampal slices with CA3, batch 6-8 from CA1 enriched).

\section{Library preparation}

All RiboSeq libraries were prepared as previously described [28]. RiboZero Gold (Illumina, \#MRZG12324) was used to deplete rRNA for RiboSeq libraries. The purified $80 \mathrm{~S}$ monosome mRNA fragments were resolved on a 15\% Tris-borate urea gel (National Diagnostics, \#EC833), and ribosome-protected fragments between 26 and $34 \mathrm{nt}$ were recovered from the gel by the crush-soak method overnight in RNA extraction buffer $(300 \mathrm{mM}$ $\mathrm{NaOAc}$ pH5.5 $1 \mathrm{mM}$ EDTA $0.25 \%$ SDS) at $25{ }^{\circ} \mathrm{C}$ with constant horizontal rotation. The RNA was then precipitated, and the purified RNA was dephosphorylated with PNK enzyme (NEB, \#M0201S) and ligated to preadenylated adaptors (IDT) using T4RNL2Tr.K227Q ligase (NEB, \#M0351L). Reverse transcription (RT) was performed using SuperScript III (Invitrogen, \#18080-044) in a modified 1X First Strand synthesis buffer without $\mathrm{MgCl}_{2}$ (50 mM Tris-HCl, pH 8.3, $75 \mathrm{mM} \mathrm{KCl}$ ). Primers containing a $5 \mathrm{nt}$ barcode and $8 \mathrm{nt}$ unique molecular identifier (UMI) were used for RT. After RT, the RNA was hydrolyzed with a final concentration of $0.1 \mathrm{~N}$ $\mathrm{NaOH}$ for 20 min at $98{ }^{\circ} \mathrm{C}$. The cDNA was resolved on a 10\% TBU gel, and the 130-140 nt band was selected. The cDNA was eluted from crushed gel pieces overnight in DNA extraction buffer (300 mM NaCl, $1 \mathrm{mM}$ EDTA, $10 \mathrm{mM}$ Tris pH8.0) at $25{ }^{\circ} \mathrm{C}$ with constant horizontal rotation. The cDNA was circularized using CircLigase (Epicentre, \#CL4115K) and depleted of tRNA and rRNA using biotinylated anti-sense probes (IDT) and Dynabeads MyOne Streptavidin C1 (Invitrogen, \#65001). To determine the optimal number of PCR cycles, a test PCR was performed using the KAPA library amplification kit (Kapa Biosystems, \#KK2611). The final PCR product was run on an $8 \%$ TBE gel, and the 180-190 nt bands were selected. The PCR product was eluted overnight in DNA extraction buffer as described earlier.

Batch 1-5 RNA-Seq libraries were prepared using RiboZero to deplete rRNA and then fragmented with $2 \mathrm{X}$ alkaline fragmentation solution $(2 \mathrm{mM}$ EDTA, $\left.10 \mathrm{mM} \mathrm{Na}_{2} \mathrm{CO}_{3}, 90 \mathrm{mM} \mathrm{NaHCO}{ }_{3}, \mathrm{pH} \sim 9.3\right)$ at $95^{\circ} \mathrm{C}$ for 15 min to yield $\sim 140 \mathrm{nt}$ fragments. The fragmented RNA was separated on a $10 \%$ TBU gel, and those migrating between 100 and $150 \mathrm{nt}$ were selected. RT products were separated on a $10 \%$ TBU gel, and the 200-250 nt band was selected. The final PCR product was run on an $8 \%$ TBE gel, and the 260-300 bp band was selected. The size distribution of the final libraries was confirmed by Fragment Analyzer (UMMS Molecular Biology Core). Batch
6-8 RNA-Seq libraries were prepared using NEXTflex poly(A) beads (Bioo Scientific, \#512980) and the NEXTFlex Rapid Directional qRNA-Seq kit (Bioo Scientific, \# NOVA-5130-03D) according to manufacturer's instructions.

The final libraries were purified using AMPure XP beads (Beckman Coulter, \#A63880). Purified libraries were quantified by qPCR using KAPA Library Quantification kit (Kapa Biosystems, \#KK4835) and were pooled to equimolar ratios. Libraries were sequenced on a NextSeq 500/550 High Output Kit v2 (Illumina, \#FC-404-2005) as 75 bp single-end runs on a NextSeq500 sequencer.

\section{RiboSeq pipeline, read mapping, and differential expression analysis}

The individual samples were separated based on $8 \mathrm{nt}$ barcodes using custom scripts. Cutadapt (1.7.1) was used to remove the adapter (TGGAATTCTCGGGTG CCAAGGAGATCGGAAGAGCGGTTCAGCAGG AATGCCGAGACCG). The reads were then uploaded to the UMass Bioinformatics Core Dolphin platform for mapping (https://www.umassmed.edu/biocore/ introducing-dolphin/). The reads were quality filtered using Trimmomatic (0.32) and then mapped to the rRNA and tRNA reference using bowtie2 (2.1.0). The rRNA and tRNA unmapped reads were kept and then mapped to mm10 using TopHat2 (2.0.9). Only uniquely mapped reads were kept, and PCR duplicates were marked based on UMI sequence and a custom script. SAMtools (0.019) was used to keep uniquely mapped reads without duplicates. For gene-level quantification of RiboSeq, RSEM/1.2.11 was used to align cleaned, unique reads to the RefSeq mm10 CDS with the first and last $30 \mathrm{nts}$ removed to avoid quantifying initiation and termination peaks. For RNA-seq libraries, reads were aligned to the entire mm 10 transcriptome. Counts from RSEM were batch-corrected using the Combat function in sva (3.24.4). Batch-corrected counts were used for DESeq2 (1.26.0) to identify differentially expressed genes for RNA and RiboSeq libraries. To validate our $T s c 2^{+/-} / \mathrm{WT}$ differential expression results from Fig. 1, we performed 4990 random permutations of the sample labels without repeating the sample order. Differential expression was performed with these randomized sample labels using DESeq2 with identical parameters, design formula (genotype treatment), and statistical cutoff $($ FDR $<0.1)$ as the real samples. The results of the randomizations are depicted in the histograms in Additional file 1: Fig S1. The majority of the analysis identified zero differentially expressed genes, and the randomizations yielded a mean of 14 DE RNAs and 11 DE RPFs, which is similar to the expected false positives from a 


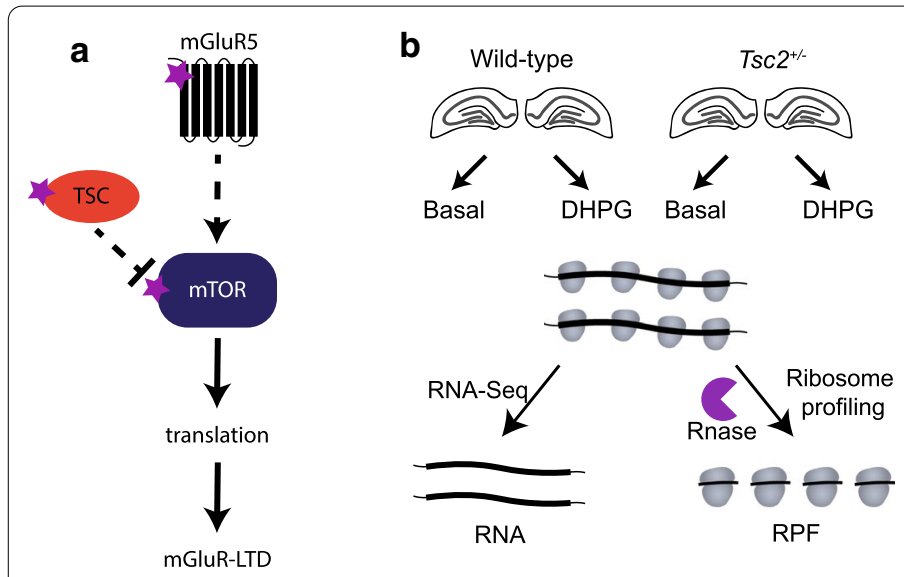

d

Tsc2+-MT DE RNA (FDR < 0.1, DE= 109)

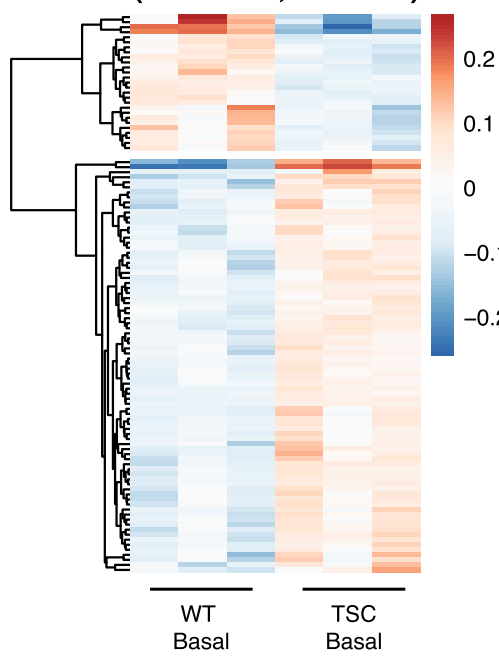

Tsc2+-NWT DE RPF

$(F D R<0.1, D E=99)$

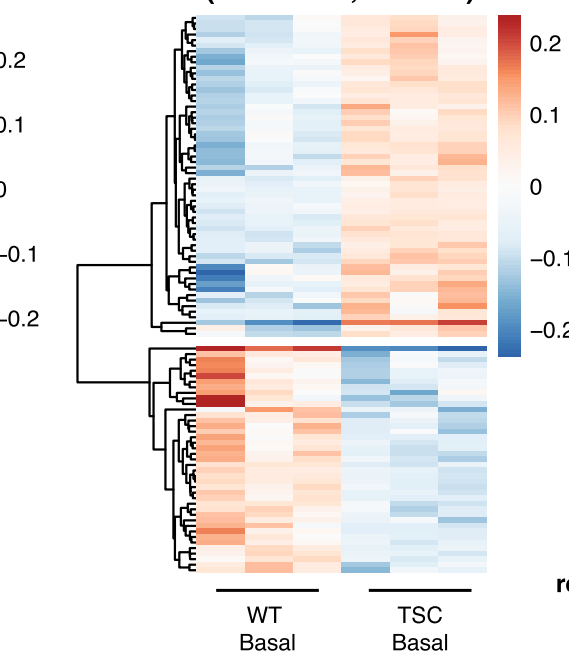

C

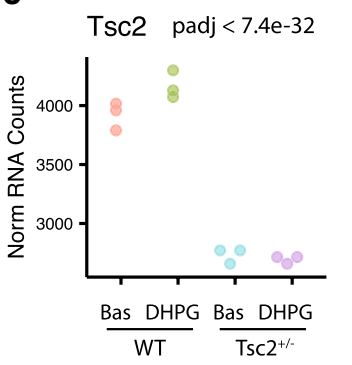

Tsc2 $\operatorname{padj}<2.4 \mathrm{e}-22$

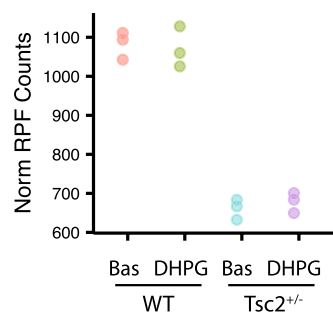

f

Enriched in Tsc2 ${ }^{+-}-\mathrm{WT}$ Up RPFs

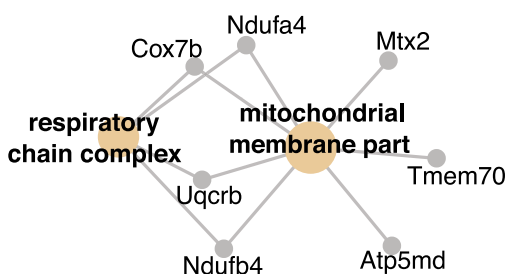

dendrite

membrane

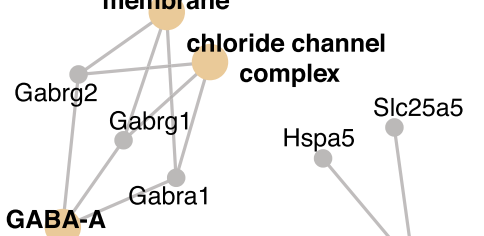

receptor complex

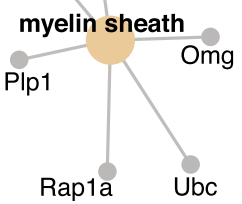

Fig. 1 Ribosome profiling of Tsc2 ${ }^{+/-}$hippocampal slices reveals RNA and RPF dysregulation. a Salient features of TSC and mGluR5 signaling. mTOR indirectly receives negative and positive signals from TSC and mGluR5, respectively. mTOR promotes translation required for mGluR5-dependent long-term depression (LTD). Dashed and solid lines indicate indirect and direct effects, respectively. The purple stars indicate SFARI autism genes. b Schematic depicting the experimental design for RNA-Seq and ribosome profiling from CA1-enriched hippocampal slices of TsC2 ${ }^{+/-}$and wild-type littermate controls. Slices were treated with DHPG (100 $\mu \mathrm{M}, 5 \mathrm{~min})$ or artificial cerebrospinal fluid (vehicle) and then processed for RNA-Seq and ribosome profiling. Slices from six mice were pooled per genotype and condition, and three biological replicates were performed. RPF, ribosome-protected fragment. c Normalized count plots of RNA and RPF levels for Tsc2 are shown (Wald-test for genotype comparison with BH correction. RNA: log2FC - 0.52, padj< $7.4 \mathrm{e}-32$; RPF log2FC -0.70, padj < 2.4e-22) $\mathbf{d}$, e Heat maps of mRNAs (d) and RPFs (e) altered in Tsc2 $2^{+/-}$ compared to WT under basal conditions. Relative gene expression is shown, and each column represents a biological replicate $(n=3)$ for RNA $(\mathrm{DE}=109, \mathrm{FDR}<0.1)$ and RPFs $(\mathrm{DE}=99, \mathrm{FDR}<0.1)$. f GO term enrichment of upregulated RPFs in $T s 2^{+/-}($padj $<0.05)$

FDR $<0.1$ of our real data (11 false positives/109 DE RNAs and ten false positives/99 DE RPF). Normalized RNA and RPF counts from DESeq2 were used for generating plots. anota2seq (1.0.1) was used to calculate expression changes in TE $(\log 2 \mathrm{FC})$ using the relative log expression (RLE) for normalization.

\section{GO term, GSEA, and gene overlap analysis}

GO term enrichment was performed using the R package clusterProfiler (3.14.0) [29]. For GSEA, mRNAs were ranked by their log2FoldChange and $p$ value from DESeq2 output. Statistical significance was corrected with Benjamini-Hochberg, and terms with $p$ adj $<0.05$ 
were considered enriched. Gene overlap analysis was performed using the $\mathrm{R}$ package GeneOverlap (1.22.0). The genes past filtering from DE analysis were used as the background.

\section{Transcript feature analysis}

Gene features were obtained from the UCSC Genome Browser using RefSeq annotations. For downstream transcript analysis, the most abundant isoform from the RSEM output of the RNA-Seq libraries was selected. ViennaRNA/RNAFold $(2.1 .6 \mathrm{~h})$ was used to calculate the minimal free energy of the $5^{\prime}$ UTR.

\section{Hippocampal TE calculation and rolling mean plots}

The average CA1 translational efficiency (TE) was calculated by taking the ratio of RPF to RNA reads per kilobase per million mapped reads (RPKM) from three wild-type basal samples aligned to the mm10 CDS reference without the initiation and termination peak. For consistent TE calculations, mapping to the CDS for RPF and RNA was used, and transcripts longer than $300 \mathrm{nt}$ and RPKM $>5$ were included.

\section{Quantification and statistical analysis}

An unpaired Wilcoxon rank-sum test with Bonferroni correction was used to compare means between groups unless indicated otherwise in the text and figures. A one-tailed Kolmogorov-Smirnov test was used as shown in Fig. 2c. An unpaired t test was used for quantification of western blots in Fig. $3 \mathrm{c}$ and Additional file 1: Fig S2B, C. Western blot results in Fig. 6a-c were analyzed with a two-way, repeated-measures ANOVA. Factors are DHPG treatment and genotype, and repeated measures are basal and DHPG levels in the same mouse. $n=\#$ mice. Post hoc Sidak's multiple comparison test was performed to determine statistical significance for specific comparisons, reported in the figure. For analysis of LTD results in Fig. 6f, g, we performed a linear mixed-effects model (LMM) statistic, whereby the variability due to random effects (animal, slice) was taken into account, allowing for direct

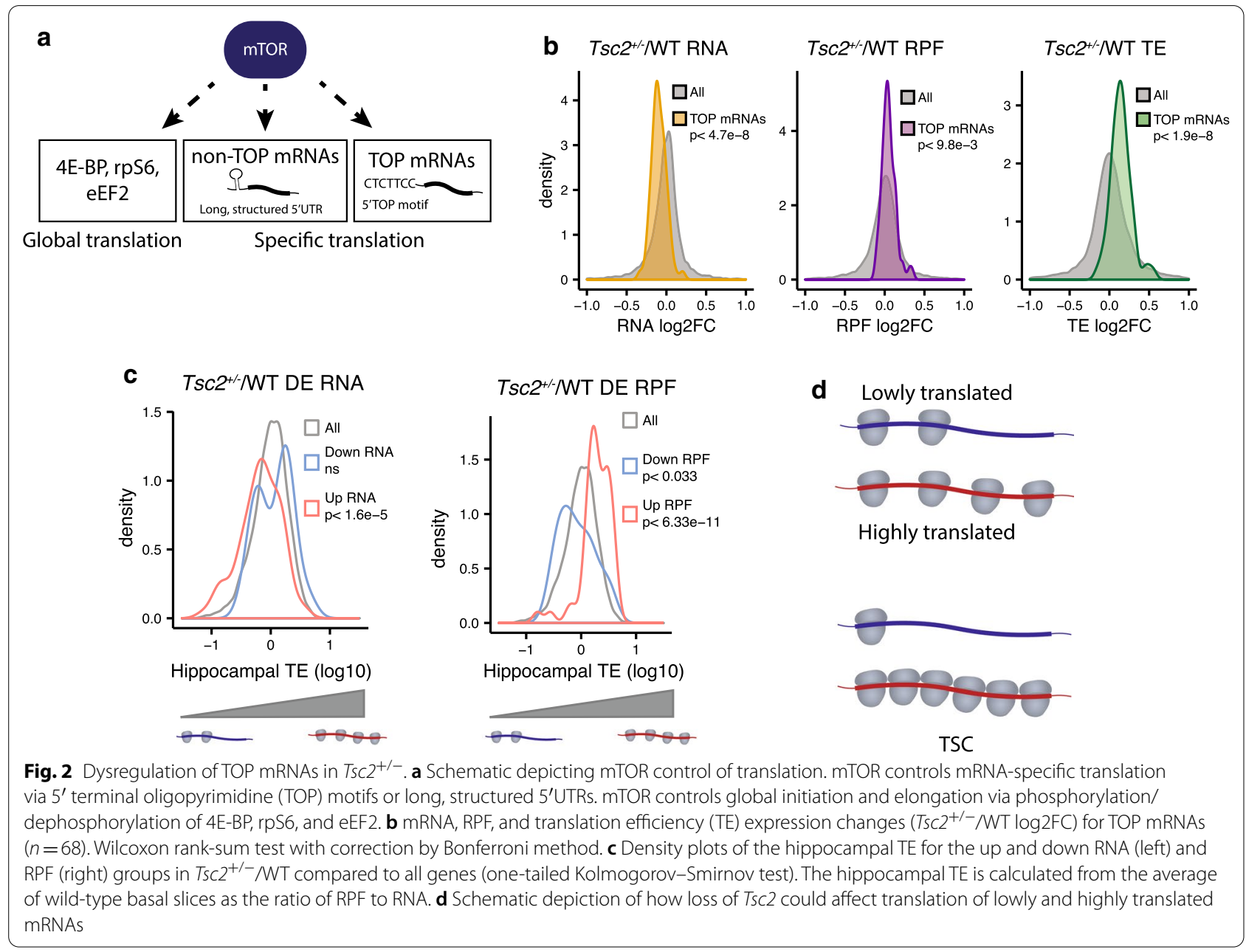



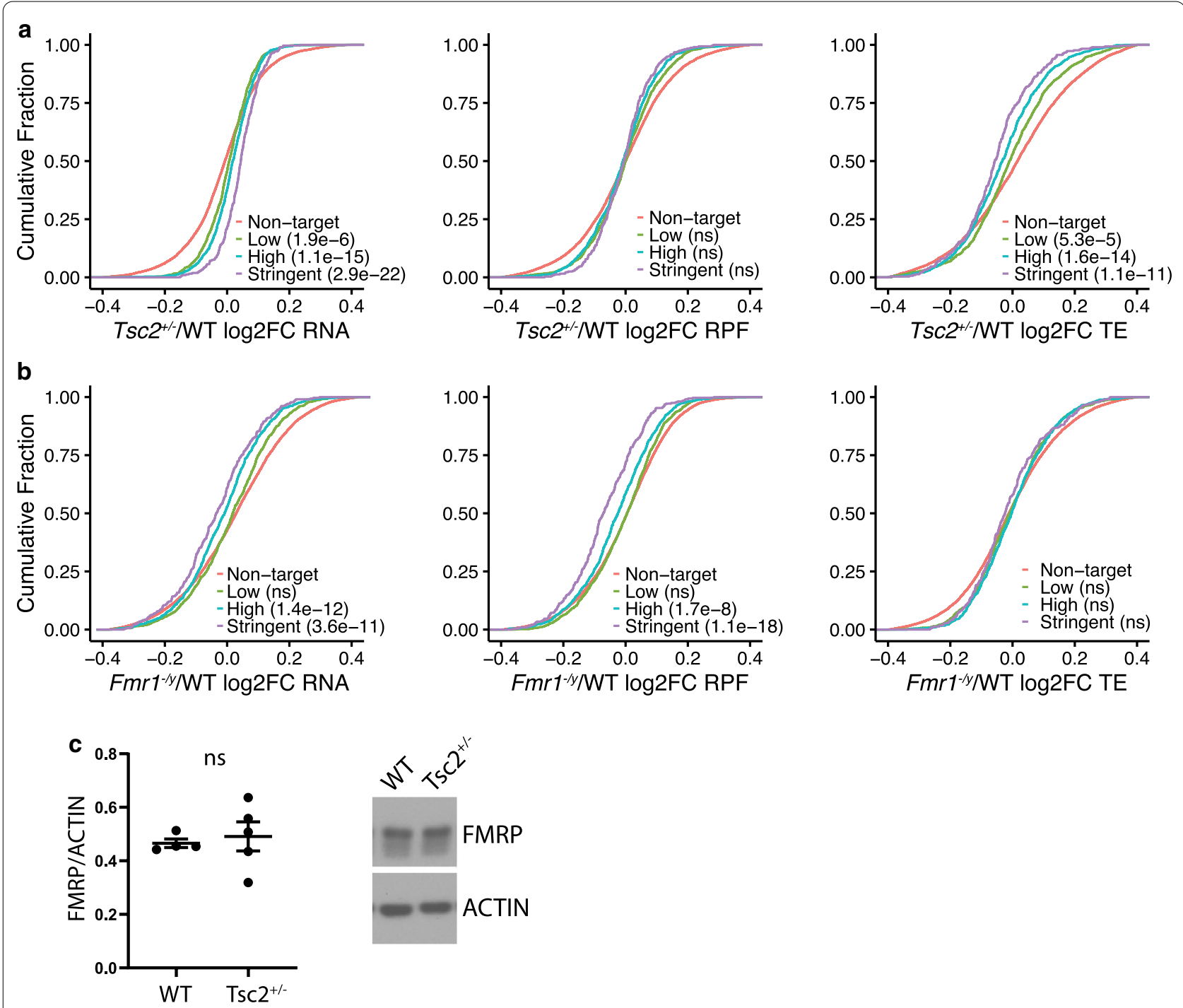

Fig. 3 FMRP binding targets are regulated by TSC and FMRP in opposing directions. a Empirical cumulative distribution function (ECDF) of RNA (left), RPF (middle), and TE (right) expression changes in TsC2 ${ }^{+/-}$WT (log2FC) of FMRP binding targets compared to nontarget mRNAs. FMRP binding targets are stratified into three groups based on binding stringency. Wilcoxon rank-sum test with correction by Bonferroni method. $p$ values are indicated in the plot legend. $\mathbf{b}$ ECDF of RNA (left), RPF (middle), and TE (right) expression changes in Fmr1 $1^{-1 y} / \mathrm{WT}$ (log2FC) of FMRP targets compared to nontarget mRNAs. Data of hippocampal slices of $\mathrm{Fmrl}^{-1 / y}$ mice from Shah et al. [76]. Wilcoxon rank-sum test with correction by Bonferroni method. $p$ values are indicated in the plot legend. $\mathbf{c}$ The levels of FMRP as determined by western blots of fresh, whole hippocampus from wild-type and $T s 2^{+/-}$littermate. $n=5$ animals, $n s$ nonsignificant, unpaired $t$ test

measurement of genotype and/or treatment effects [30]. Mixed-effects models were fitted using IBM SPSS package. Fixed factors were genotype, treatment, genotype "treatment; repeated/random effects were slices nested within subject (mouse).

\section{Code availability}

Custom scripts for generating plots will be available upon request to the lead contact.

\section{Results}

\section{Translational profiling of the TSC hippocampus}

The GTPase activating protein tuberous sclerosis complex (TSC) is an upstream but indirect repressor of mTOR, a kinase which forms the multi-subunit complex mTORC1 to stimulate translation in response to a number of signaling inputs including activated group I metabotropic glutamate receptors (e.g., mGluR5). mGluR5 activation leads to long-term depression (LTD), a protein synthesis-dependent form of synaptic plasticity 
frequently examined at hippocampal Schafer collateralCA1 synapses (Fig. 1a) [12]. TSC is a neurodevelopmental disorder characterized by autism, epilepsy, and intellectual disability, and to study the role of TSC2 in synaptic plasticity, we generated mice lacking one copy of the $T s c 2$ gene, which will henceforth be referred to as the $T s c 2^{+/-}$mouse.

To detect subtle changes in translation, we turned to ribosome profiling, a high-resolution and unbiased whole transcriptome method for determining the number and positions of ribosomes on mRNA. When combined with RNA-seq, the ratio of ribosome occupancy over input mRNA yields translational efficiency (TE) [23]. Although TE is often used as a proxy for protein synthesis, changes in TE may not always reflect protein output when ribosome elongation or mRNA levels are altered. Hippocampal slices, some of which were treated with dihydroxyphenylglycine (DHPG) for $5 \mathrm{~min}$ to induce LTD, were lysed in the presence of cycloheximide to prevent ribosome run-off, digested with RNase, and the $80 \mathrm{~S}$ monomers containing $\sim 30 \mathrm{nt}$ ribosome-protected fragments (RPFs) were collected by ultracentrifugation (Fig. 1b). The isolated RPFs were appended with primers for library construction and sequencing ( $>10$ million uniquely mapped reads; libraries were strongly correlated with each other; Pearson's $R>0.99$ ).

$T s c 2$ was the top differentially expressed mRNA at both the RNA and RPF levels, confirming the validity of our approach (Fig. 1c). Figure 1d, e shows heat maps of the RNAs and RPFs, respectively, from the hippocampal slices of the $T s c 2^{+/-}$mice and their littermate controls under basal (unstimulated) conditions that pass a significance cutoff of FDR $<0.1$. The differentially expressed (DE) RNAs and RPFs altered in $T s c 2^{+/-}$had little overlap with each other $(n=5)$. The ratio of RPF to RNA yields translational efficiency (TE), but because changes in TE do not distinguish between RPF and RNA effects and because few RNAs passed statistical cutoff for changes in TE $(\log 2 \mathrm{FC})$, we considered RNA and RPF changes separately.

We observed enrichment for terms related to the GABA-receptor complex, mitochondrial membrane, and myelin sheath for RPFs that were Upregulated in the $T s c 2^{+/-}$brain (Fig. 1f, see Additional file 2: Table S1). No GO terms were enriched in the downregulated RPF group, likely due to the small number of genes. An altered excitatory/inhibitory balance is frequently observed in ASD, and the increased translation of GABA-Arelated proteins may be a compensatory response to the increased hyperexcitability observed in $T s c 2^{+/-}$mice [31-35]. Altered translation of mitochondrial membrane proteins is consistent with the role of mTOR-dependent translational control of mitochondrial-related mRNAs and reports of mitochondrial dysfunction in Tsc2-deficient cultured neurons [36, 37]. The abnormalities in myelination in TSC patients and function of TSC/mTOR in myelination and function of oligodendrocytes [38, 39] may be due to translational control of myelin sheath proteins.

\section{Dysregulation of $5^{\prime}$ terminal oligopyrimidine (TOP) mRNAs} The major downstream substrate of TSC is mTOR, which controls specific and global translation (Fig. 2a). mRNAs that contain a $5^{\prime}$ terminal oligopyrimidine motif (TOP), which generally encode components of the translational machinery, and mRNAs with long, structured $5^{\prime}$ UTRs are especially sensitive to mTOR regulation [40-43]. $\mathrm{TSC} / \mathrm{mTOR}$ also regulates general translation through phosphorylation/dephosphorylation events such as those that occur on 4E-BP, eIF4B, rpS6, and eEF2 [43-46]. Elevated mTOR signaling contributes to various behavioral and synaptic plasticity phenotypes in the brains of TSC mice as evidenced by their rescue by treatment with the mTOR inhibitor rapamycin [2, 24]. We found that the TOP mRNAs had mildly increased RPFs, but as a group, they had increased TE that is primarily driven by decreased RNA levels (Fig. 2b). mTOR can also promote initiation of non-TOP mRNAs that have long, structured $5^{\prime}$ UTRs via eIF4E and eIF4A [40]. However, we find no difference in the $5^{\prime} \mathrm{UTR}$ lengths or predicted secondary structures in the up- and downregulated RPFs (Additional file 1: Fig S2A). To assess whether signaling events usually associated with mTOR activation occur in $T s c 2^{+/-}$hippocampus, we examined levels of phosphorylated mTOR, rpS6, and 4E-BP1. We also performed western blots for two TOP mRNAs, RPL4 AND RPS25, as well as LARP1, a TOP RNA-binding protein [47, 48]. Changes in the basal levels of these proteins are undetectable in the TSC hippocampus, and there is only a trend toward increases in phospho-4E-BP1 (Additional file 1: Fig S2B, C), similar to previous reports [35].

Because we did not identify specific mRNA features in the mRNAs and RPFs dysregulated in $T s c 2^{+/-}$mice, we wondered whether the mRNA-specific dysregulation may arise by altered global protein synthesis put forward by the "ribosome concentration" hypothesis from Mills and Green [49]. Their "ribosome concentration" hypothesis proposed that mRNAs with different rates of initiation respond bidirectionally to subtle changes in global translations from altered ribosome levels. Although we do not have evidence of altered mTORC1-rpS6 signaling or ribosome concentrations in $T s c 2^{+/-}$mice, we used this model as an intellectual framework for interpreting our results. We approximated initiation by calculating TE from wildtype basal slices, which will be referred to as hippocampal TE. The Up RNAs in $T s c 2^{+/-}$had a low hippocampal 
TE compared to all (Fig. 2c, left). We find that mRNAs with Down RPFs in $T s c 2^{+/-}$had a low hippocampal TE, and the Up RPFs had a high hippocampal TE compared to all (Fig. 2c, right). These results suggest that in $T s c 2^{+/-}$ mice, poorly translated mRNAs are reduced even further, while highly translated mRNAs become more robustly translated (Fig. 2d). Finally, Additional file 1: Figure S1D shows transcript features that correlate with hippocampal TE. Consistent with another study, hippocampal TE is correlated with GC content of the CDS, 3'UTR, and $5^{\prime} \mathrm{UTR}$ and also CDS length [50].

\section{FMRP binding targets are altered in opposite directions in TSC and FXS mice}

Loss of the RNA-binding protein Fragile X Mental Retardation Protein (FMRP) leads to Fragile X Syndrome (FXS). FMRP and TSC2 bidirectionally regulate mGluRLTD and behavior, suggesting they coregulate similar mRNAs. In support of this, a combined TSC/FXS mouse rescues deficits in synaptic plasticity and learning to wild-type levels compared to the single mouse mutants [2]. Given the rescue paradigm of the FXS and TSC mouse, we determined whether FMRP targets are altered in $T s c 2^{+/-}$mice. We used a list of FMRP targets identified by CLIP (cross-link and immunoprecipitation) in CA1 pyramidal neurons, where FMRP targets were stratified into three groups based on binding strength: stringent $(n=327)$, high $(n=938)$, and low $(n=1330)$ binding targets [51]. FMRP targets are enriched $(p<5.3 \mathrm{e}-8)$ in the mRNAs altered in TSC (from Fig. 1d), and as a group, they have decreased TE that is driven by increased RNA levels that correlate with stringency of FMRP binding (Fig. 3a). FMRP targets have no change in RPFs in TSC (Fig. 3a, middle). Interestingly, the FMRP targets are decreased in the FXS mouse $\left(F m r 1^{-1 /}\right)$, which is also correlated with stringency of FMRP binding (Fig. 3b) [51-54]. Because FMRP binding targets were dysregulated in $T s c 2^{+/-}$, we determined whether FMRP protein levels were altered in $T s c 2^{+/-}$. FMRP levels were unaltered in $T s c 2^{+/-}$(Fig. 3c), suggesting that the increased RNA levels of FMRP targets in $T s c 2^{+/-}$mice are not caused by altered FMRP levels. This bidirectional change in FMRP target mRNAs could be linked to the TSC/FXS mouse rescue paradigm and reveals a complex interaction between TSC2 and FMRP in steady-state regulation of mRNAs in the hippocampus.

\section{DHPG-induced mGluR signaling induces a rapid RNA and translational response}

mGluR-LTD is regulated by TSC, so to determine the identity of mGluR-stimulated translation of specific RNAs during LTD, we performed ribosome profiling and RNA-seq on hippocampal slices following $5 \mathrm{~min}$ of DHPG treatment $[12,18,22]$. Figure $4 a, b$ shows that RNA $(\mathrm{DE}=241, \mathrm{FDR}<0.01)$ and $\mathrm{RPF}(\mathrm{DE}=212$, FDR $<0.05)$ levels undergo rapid changes in response to the mGluR1/5 agonist DHPG. DHPG-responsive RNAs and RPFs largely did not overlap $(n=5)$. Our DHPG-responsive translational changes in area CA1 do not overlap with those identified by polysome RNA-seq in cultured cortical neurons, which we attribute to different sample types and methodologies [21]. Gene set enrichment analysis reveals that GO terms related to $G$ protein-coupled receptor activity, synaptic membranes, and the endoplasmicreticulum membrane are enriched in the upregulated RNA and RPFs, and helicase activity is enriched in the downregulated RNAs and RPFs (Additional file 1: Fig S3A, B, Additional file 3: Table S2). Some GO terms are unique to the RNA or RPF changes. For example, the RNAs upregulated following DHPG are enriched for transcription-related functions, and the DHPG translational response is enriched for terms related to the lysosome. Some examples of DHPG-responsive mRNAs and RPFs that are high confidence SFARI autism genes (https://gene.sfari.org, score 1 or 2) are shown in Fig. 4c for Shank3, Dlg4, and Ache. Dlg4 mRNA encodes PSD-95, which has increased mRNA and protein levels following DHPG-mGluR signaling [55]. Nrsn1 mRNA encodes a neuron-enriched regulator of vesicle transport, which is translationally repressed $5 \mathrm{~min}$ after a hippocampus-dependent learning task [56]. Translational repression of Nrsn1 mRNA during learning may be a requirement for this cognitive process as evidenced by the observation that its overexpression leads to deficits in object location and contextual fear memory. Because we did not observe immediate early genes (c-fos, $A r c$, and Egrl) in our list of differentially expressed RNAs, we infer that the rapid changes in RNA levels are likely due to alterations in RNA destruction and not transcription.

Because FMRP is regulated by and required for mGluR1/5 signaling to translation [57-60], we determined whether FMRP mRNA targets are responsive to this form of synaptic plasticity. FMRP binding targets are enriched in the DHPG-responsive DE RNAs $(p<1.8 \mathrm{e}-44)$ and RPFs $(p<5.0 \mathrm{e}-9)$. As a group, the FMRP targets have increased RNA levels following mGluR signaling that correlate with stringency of FMRP binding (Fig. 4d, left). FMRP targets have decreased RPFs following synaptic stimulation, and an overall decrease in TE that correlates with FMRP binding stringency (Fig. 4d, middle and right). Therefore, mGluR stimulation regulates translation and RNA levels of FMRP target mRNAs. 


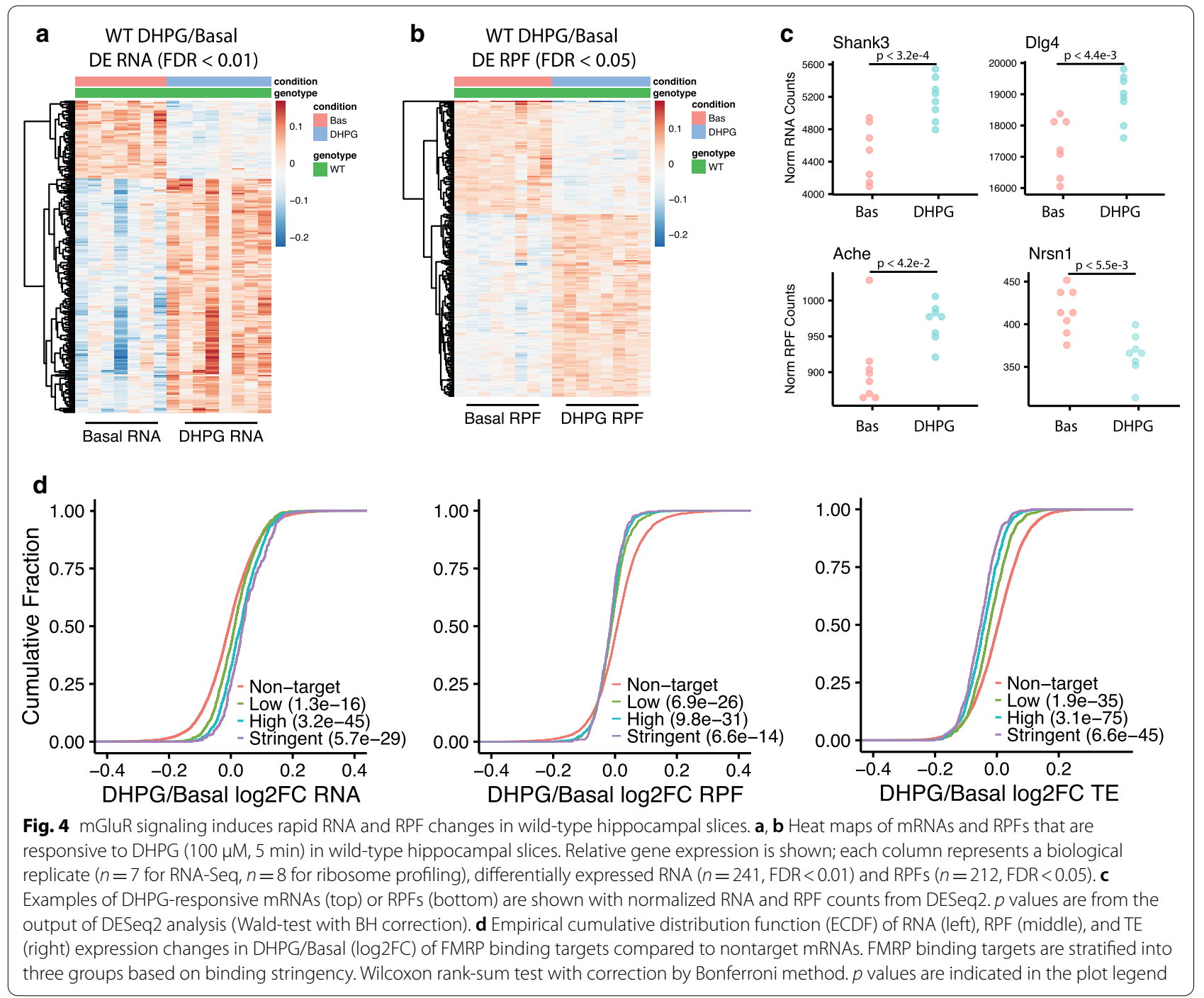

\section{mGluR-induced translational dysregulation in TSC}

$T s c 2^{+/-}$mice have deficient mGluR-LTD, so we determined whether the RNA and translational response after DHPG is altered. We lacked sufficient statistical power to detect genotype-dependent alterations in the DHPG response in $T s c 2^{+/-}$slices with their littermate controls. Instead, we compared the genes altered in $T s c 2^{+/-}$ (Fig. 1d, e) and the DHPG-responsive genes in wild type (Fig. 4a, b) and found a statistically significant overlap for the RNAs $(n=23, p<4.3 \mathrm{e}-21)$ and RPFs $(n=20$, $p<3.8 \mathrm{e}-18$ ) (Fig. 5a, b; Additional file 4). Interestingly, 16 of the 23 RNAs that overlap between $T s c 2^{+/-}$and mGluR1/5-stimulated are FMRP mRNA targets (e.g., Kif5a, Snph, Nisch, and Slc25a23). No GO terms were enriched in the overlapping list, likely due to the small number of genes. Heat maps of the overlapped mRNAs and RPFs in Fig. 4a, b show that most of the upregulated mRNAs and RPFs in unstimulated $T s c 2^{+/-}$slices appear to have a deficient DHPG response compared to wild-type slices (Fig. 5c, d). Our results suggest that some mRNAs and RPFs that are elevated in unstimulated $T s c 2^{+/-}$slices cannot respond to DHPG.

\section{Signaling to eukaryotic elongation factor 2 is altered in the $\mathrm{TsC2}^{+/-}$hippocampus}

The sequencing results of the $T s c 2^{+/-}$slices under basal and DHPG conditions suggest there is a deficiency in the ability of DHPG to regulate translation in the $T s c 2^{+/-}$ mice. To determine whether mGluR1/5 signaling to translational control was altered in $T s c 2^{+/-}$, hippocampal slices were treated with DHPG followed by western blotting for several well-established downstream substrates of mGluR activation. Figure 6a, b shows that under basal conditions, phosphorylated (phospho) ERK and AKT were unaffected by loss of one allele of Tsc2. In response 


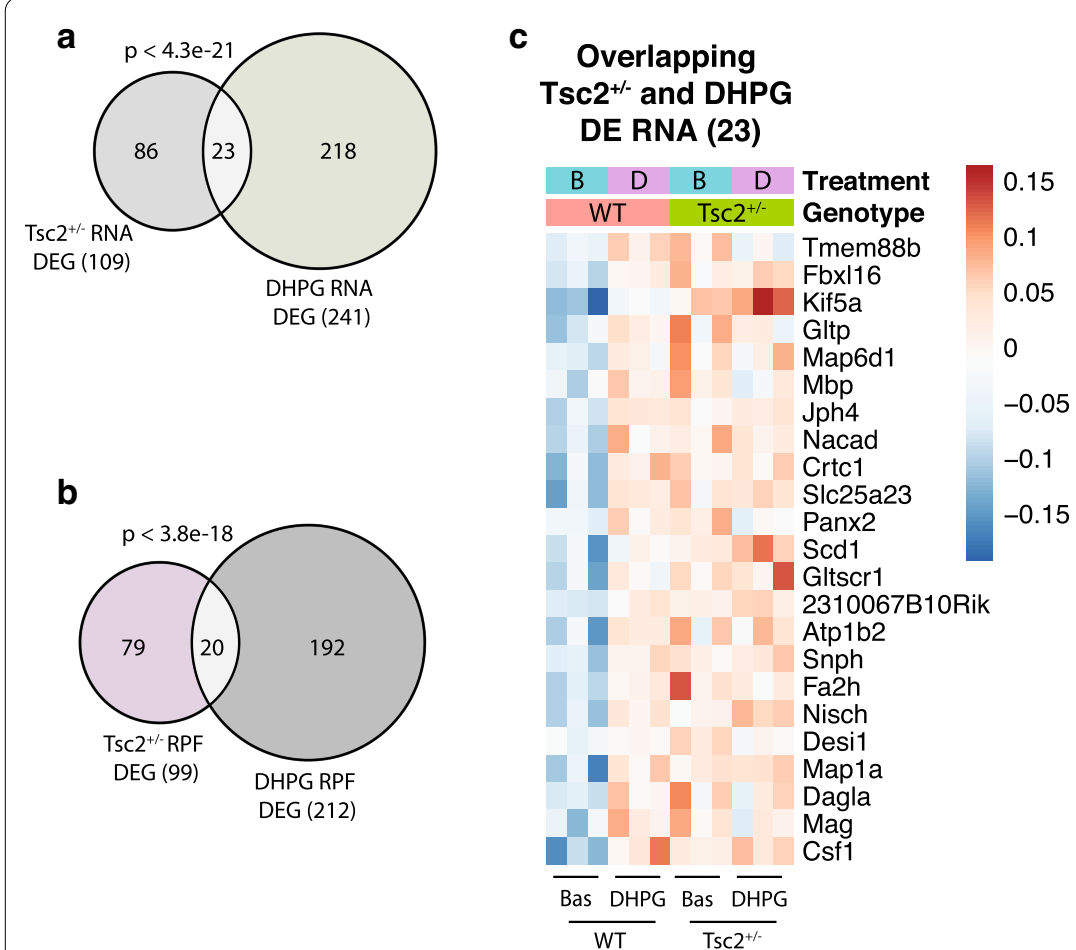

\section{d \\ Overlapping Tsc2+/ and DHPG DE RPF (20)}

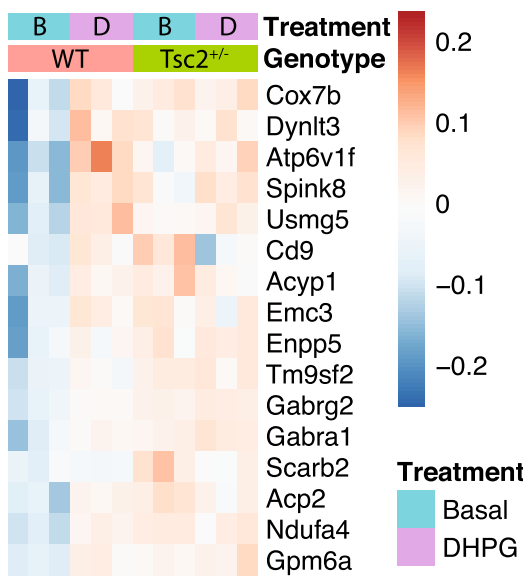

Fig. 5 Some mRNAs altered in unstimulated $T s c 2^{+/-}$slices do not respond to DHPG-mGluR signaling. $\mathbf{a}$, $\mathbf{b}$ Venn diagrams showing overlap between $\mathrm{TsC}_{\mathrm{c}}{ }^{+/-}$dysregulated RNAs and DHPG-responsive RNAs (a) and Tsc2 ${ }^{+/-}$dysregulated RPFs and DHPG-responsive RPFs (b). Gene overlap test was performed using Fisher's exact test and a background size of expressed RNAs used in DE analysis. $\mathbf{c}$, $\mathbf{d}$ Heat maps of mRNAs (c) and RPFs (d) that overlap from (a) and (b). Relative gene expression is shown, and each column represents a biological replicate of littermates $(n=3)$

\section{(See figure on next page.)}

Fig. 6 Deficient mGluR1/5-regulated signaling to translation elongation suppresses mGluR-LTD in TsC2 ${ }^{+/-}$mice. a-c The levels of phosphorylated (P) and total (T) ERK (a), AKT (b), and eEF2 (c) in CA1-enriched hippocampal slices from wild-type and TsC2 ${ }^{+/-}$mouse littermates as determined

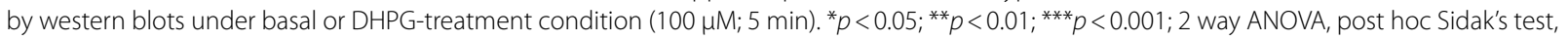
$n=11-19$ mice/genotype, 4-5 slices per drug condition/mouse. Asterisks below brackets indicate an effect of DHPG on phosphoprotein levels within genotype. Asterisks above brackets indicate effects between genotypes. $\mathbf{d}$ Schematic depicting that global slowing of ribosome movement through eEF2 phosphorylation or low dose cycloheximide (CHX) may promote protein synthesis of lowly translated mRNAs while inhibiting highly translated mRNAs. e Empirical cumulative distribution function (ECDF) plot of hippocampal TE for the Up and Down RNAs (left) and RPFs (middle) in DHPG/basal compared to all genes. Right: ECDF plot of hippocampal TE for FMRP binding targets compared to non-target mRNAs. FMRP binding targets are stratified into three groups based on binding stringency. Wilcoxon rank-sum test with correction by Bonferroni method. $p$ values are indicated in the plot legend. $\mathbf{f}$ Left: Time course demonstrating that brief DHPG application ( $100 \mu \mathrm{M} ; 5$ min) induces long-term synaptic depression (LTD) of field (f) EPSP slopes in WT hippocampal slices that is unaffected by low doses of cycloheximide (45-60 min pretreatment, $75 \mathrm{nM} ; n=12$ and 15 slices; 6-7 mice/condition). Right: In $\mathrm{TsC2}^{+/-}$mice pretreatment with low-dose cycloheximide enhances LTD magnitude ( $n=10$ and 15 slices; 7 mice/condition). Plotted are group averages of fEPSP slope (mean \pm SEM) normalized to pre-DHPG baseline as a function of time. Inset: Example fEPSP from baseline and during LTD (55-60 min post DHPG). Scale $=0.5 \mathrm{mV} / 10 \mathrm{~ms}$. $\mathbf{g}$ LTD magnitude, measured at 55-60 min post-DHPG application in vehicle and cycloheximide in each genotype. There is a significant interaction between cycloheximide and Tsc2 genotype $(F(1,47)=5.197, p<0.05 ; \mathrm{LMM})$. With post hoc pairwise comparisons, there is a strong trend for cycloheximide to enhance LTD in TsC2 ${ }^{+/-}$mice (veh: $83 \pm 2 \%$ of baseline; cycloheximide; $75 \pm 5 \% ; n=15$ and ten slices from four mice/treatment; $F(1,23)=5.445, p=0.058 ;$ LMM) but not WT mice (veh: $74 \pm 2 \%$ of baseline; cycloheximide; $77 \pm 3 \% ; n=11$ and 15 slices from 6 and 5 mice, respectively/treatment; $F(1,24)=3.819, p=0.14 ; L M M)$. In vehicle, LTD is reduced in TsC2+/- mice in comparison with WT, but not in low-dose cycloheximide. $\left.{ }^{*} p<0.05 ;{ }^{* *} p<0.01\right)$. h Schematic showing DHPG induction of eEF2 phosphorylation in hippocampal slices. In WT slices, DHPG leads to increased levels of phospho-eEF2 through signaling to eEF2K, which presumably slows ribosome transit and promotes expression of lowly translated mRNAs required for mGluR-LTD, such as FMRP binding targets. In TSC slices, levels of phospho-eEF2 are elevated basally and correlate with increased mRNA abundance of FMRP binding targets. DHPG treatment does not further increase eEF2 phosphorylation, and $\mathrm{TsC}^{+/-}$slices may have excessive ribosome transit during mGluR signaling that contributes to deficient mGluR-LTD. 


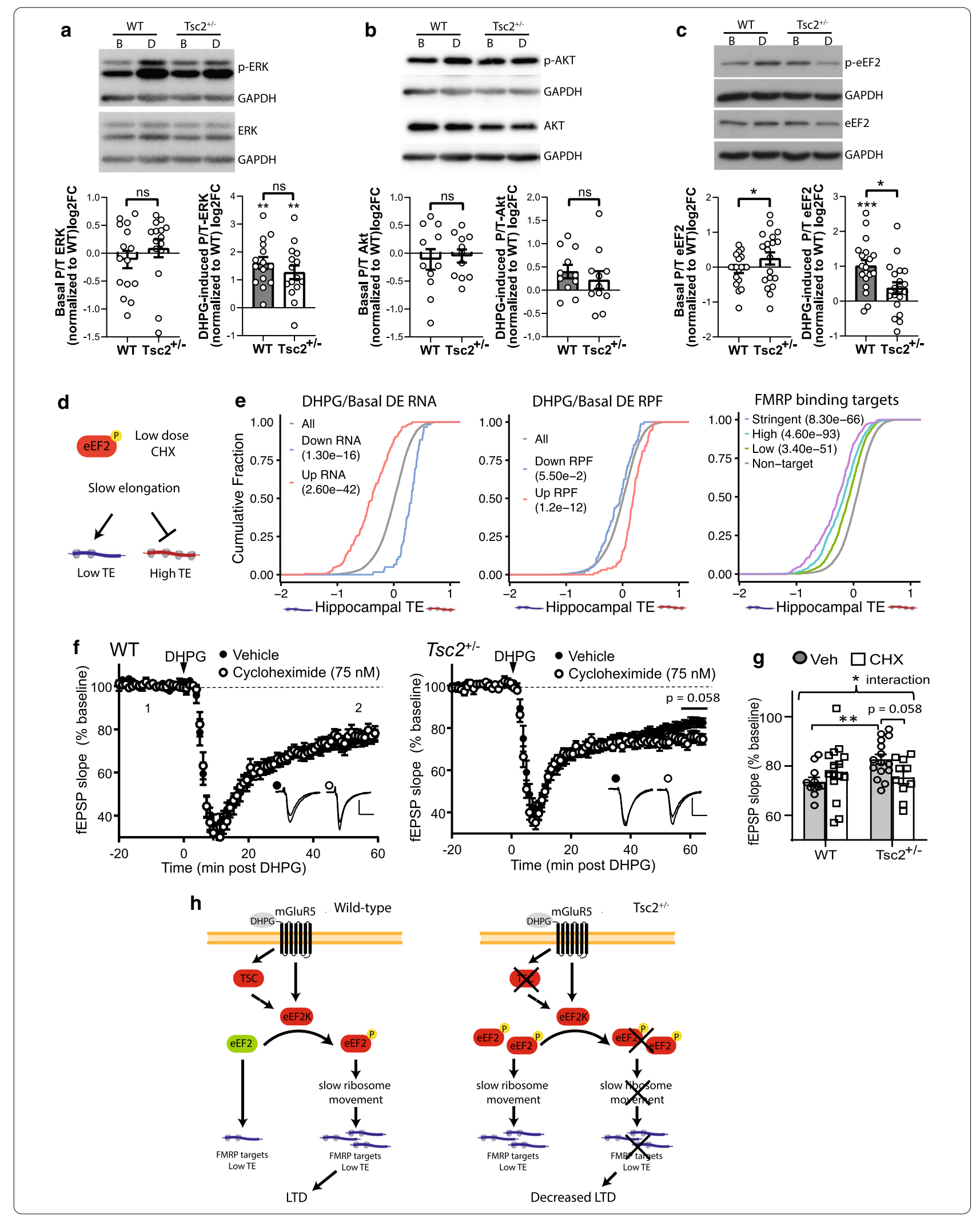


to DHPG treatment, phospho-ERK levels were similarly elevated in WT and $T s c 2^{+/-}$slices, consistent with other observations [2, 42]. DHPG did not induce phosphoAkt in either WT or $T s c 2^{+/-}$slices. Because both mTOR signaling and mGluR5 signaling regulate phosphorylation of eukaryotic elongation factor 2 (eEF2), we determined whether signaling to eEF2 could be altered. In wild-type slices, DHPG treatment robustly induces eEF2 phosphorylation (Fig. 6c). Surprisingly, $T s c 2^{+/-}$slices have elevated phospho-eEF2 basally, and DHPG treatment fails to induce eEF2 phosphorylation. Total levels of eEF2 are unchanged in $T s c 2^{+/-}$at basal $(106 \pm 6 \%$ of WT; $n=19$ mice; n.s.; 2way ANOVA, post hoc Sidak's test) and following DHPG treatment (WT 101.7 $\pm 8.02 \%$, $T s c 2^{+/-} 114.4 \pm 9.69 \%, n=19$, n.s.; 2way ANOVA, post hoc Sidak's test).

eEF2 is a GTP-dependent elongation factor that promotes transfer of peptidyl-tRNAs from the A to P site of the ribosome. Phosphorylation of eEF2 on threonine 56 prevents it from binding to ribosomes, leading to inhibition of translation elongation [61]. Somewhat counterintuitively, eEF2 kinase and its phosphorylation of eEF2 are necessary for mGluR-induced synthesis of proteins required for LTD [18]. Global inhibition of translation elongation, such as through phospho-eEF2 or cycloheximide (a chemical inhibitor of ribosome translocation), may promote synthesis of poorly translated mRNAs by liberating rate-limiting translation factors (Fig. 6d) $[18,62,63]$. Thus, we determined whether the mRNAs responsive to mGluR-LTD signaling are enriched for poorly translated mRNAs by using hippocampal translation efficiency as described earlier. We find that the Up RNA group after DHPG treatment has low basal translation efficiency compared to unchanged mRNAs and the Down mRNA group has high basal translation efficiency (Fig. 6e, left). For the translational response, the Up RPF group after DHPG-mGluR signaling has high basal translation efficiency compared to other mRNAs, whereas the Down RPF group had the same translation efficiency as the unchanged mRNAs (Fig. 6e, middle). Because DHPG-mGluR signaling may reduce ribosome translocation via phospho-eEF2, it is possible that at least some of the mRNAs with increased RPFs are associated with stalled ribosomes, in which case they would have decreased translation. Because FMRP binding targets are enriched in the DHPG-mGluR-responsive Up mRNA group, we predicted that FMRP targets are also lowly translated in the hippocampus. Indeed, FMRP binding targets have low basal translation efficiency compared to nontargets (Fig. 6e, right). Interestingly, the strength of FMRP binding negatively correlates with basal translation efficiency where the most stringent FMRP targets have lower basal translation efficiency compared to the low- and high-stringency groups. Taken together, these observations suggest that mGluR5 signaling increases expression of mRNAs that are lowly translated, such as FMRP binding targets.

Because $T s c 2^{+/-}$slices have a deficient phospho-eEF2 response to DHPG treatment, we hypothesized that ribosome elongation is excessive during $\mathrm{mGluR}$ signaling in $T s c 2^{+/-}$and may contribute to deficient mGluR-LTD. We used a low concentrations of cycloheximide (75 nM), which inhibits ribosome elongation by binding to the E-site of the ribosome to prevent eEF2-mediated translocation [64]. This concentration of cycloheximide restored mGluR-LTD in eEF2K knockout mice without affecting mGluR-LTD in WT mice [18]. We induced mGluR-LTD with DHPG in hippocampal slices and tested the effects of low-dose cycloheximide (75 nM) to inhibit ribosome elongation. While there were no main effects of genotype or treatment overall on LTD magnitude, we observed a significant interaction of genotype treatment (Fig. 6f, g). With post hoc pairwise comparisons, there was a strong trend for cycloheximide to enhance mGluR-LTD in $T s c 2^{+/-}$(veh: $83 \pm 2 \%$ of baseline; cycloheximide; $75 \pm 5 \%$ ) but not WT mice (veh: $74 \pm 2 \%$ of baseline; cycloheximide; $77 \pm 3 \%$ ). In vehicle, mGluR-LTD in $T s c 2^{+/-}$was less than WT as previously reported [2]. Our data suggest that the deficiency of mGluR1/5 induced P-eEF2 and suppression of translation elongation in $\mathrm{TsC}^{+/}$mice limits LTD magnitude and this can be reinstated by pharmacological inhibition of ribosome elongation (Fig. 6h).

\section{Discussion}

Using ribosome profiling and RNA-seq, we identified changes in ribosome footprint number and RNA levels in $T s c 2^{+/-}$hippocampal slices and following mGluR-LTD. Our experimental design captures changes from multiple cell types, which is reflective of the tissue complexity of the hippocampus. We found translation of myelin-related mRNAs increased in $T s c 2^{+/-}$mice, which are predominantly found in oligodendrocytes. The tissue complexity may explain why we did not capture translation of known DHPG-induced mRNAs Arc and Map1b, which are translated following DHPG treatment in dendrites of excitatory neurons [22, 65].

In complete loss of TSC1 or TSC2 models, there is clearly excessive mTOR activity that leads to widespread changes in translation [66]. However, mTOR hyperactivation is not always as obvious in Tsc1 and Tsc2 heterozygote models in the brain [35]. Using a $T s c 2^{+/-}$model, we do not observe signs of mTOR hyperactivation by western blot of phosphorylated mTOR, 4E-BP1, and rpS6 in the hippocampus. Instead, we find evidence of disrupted global mRNA translation by increased TE of TOP 
mRNAs and elevated levels of phosphorylated eEF2. The TOP mRNAs, the most well-studied TSC/mTOR translational targets, have increased TE from predominantly decreased RNA and increased RPF levels. The decreased RNA levels of the TOP mRNAs are surprising, given that one predicts increased expression of TOP mRNAs from mTOR hyperactivity from loss of TSC2. There could be two possibilities to explain this observation. This may be an example of translational buffering to maintain homeostasis of proteins encoded by TOP mRNAs, where RNA levels decrease to offset the increase in ribosome occupancy $[67,68]$. Alternatively, the decreased RNA levels of TOP mRNAs may be reflective of the altered signaling to phospho-eEF2, which is increased and opposite of the direction that is predicted by activation of mTORC1S6K-eEF2K signaling in $T s c 2^{+/-}[45,46]$. The increased in basal phosphorylated eEF2 in $T s c 2^{+/-}$mice may occur via other effectors to eEF2K, such as increased intracellular $\mathrm{Ca} 2+$ and mGluR1/5 signaling $[18,69]$. Our finding of increased basal levels of phospho-eEF2 is consistent with the observation of decreased protein synthesis rates in hippocampal slices of $T s c 2^{+/-}$mice [2]. Our study suggests a more complex role of translational dysregulation in the $T s c 2^{+/-}$hippocampus outside of simple mTORC1rpS6 hyperactivation. Given the many downstream substrates of mTOR, it is possible that some of the mRNA and translational alterations observed in $T s c 2^{+/-}$are through mTORC1-dependent processes outside of translation (e.g., autophagy, lipogenesis) or mTORC2. The small sample size for the Tsc2 genotype comparison is a limitation to our sequencing analysis, which has low power to identify differences from lowly expressed genes and small effect changes frequently observed in the brain and neuropsychiatric disorders. Our results do not rule out more dramatic, cell-type specific arising from $T s c 2$ haploinsufficiency, such as in excitatory neurons. These results also highlight the importance of maintaining appropriate gene dosage in modeling TSC and other mTORopathies such as PTEN, where only one allele is usually mutated in patients.

Because $T s c 2^{+/-}$mice have decreased protein synthesis in the hippocampus and DHPG-mGluR1/5 signaling can activate near global regulators of translation, and we used the "ribosome concentration" hypothesis as an intellectual framework for interpreting our results. This model was originally proposed to explain the seemingly mRNAspecific effects arising from ribosomopathies, a group of disorders with mutations in ribosomal proteins that manifest with cell-type-specific features. Using mathematical modeling and assuming initiation as the rate-limiting step of translation, the "ribosome concentration" hypothesis predicts that efficiently and inefficiently translated mRNAs respond bidirectionally to global decreases in translation arising from reduced levels of ribosomes, and recent experimental evidence from yeast and hematopoietic cells supports this hypothesis, albeit in a direction opposite of originally predicted $[70,71]$. Here, we observe a trend where in $T s c 2^{+/-}$mice, inefficiently translated mRNAs have decreased translation and efficiently translated mRNAs have increased translation (Fig. 2e). Surprisingly, during DHPG-mGluR1/5 signaling, the degree of translation efficiency correlates more with RNA-level changes rather than translation, and FMRP binding targets have low translation efficiency compared to all genes (Fig. 6e, left). The factors that regulate this phenomenon in $T s c 2^{+/-}$mice and DHPG-mGluR1/5 signaling remain unknown.

RNA-seq reveals that mGluR activation induces a rapid response in both increased and decreased mRNA levels. Because translation is often linked to RNA stabilization/ destabilization, this mGluR response could be linked to this process [72]. The decrease in specific mRNAs may be the result of heightened rates of degradation, which could be the consequence of mGluR-dependent upregulation of microRNAs [73]. For mRNAs that increase, there may be a constant rate of mRNA turnover in hippocampal tissue, and mGluR activation could lead to mRNA stabilization through regulation of translation or microRNA dissociation [60]. The DHPG-mGluR-responsive mRNAs are highly enriched for FMRP targets and genes encoding synaptic proteins. For example, our study and that of Zalfa et al. [55] found that levels of PSD-95 mRNA $(D \lg 4)$, an FMRP target, increase after mGluR activation in cultured neurons and are regulated by interaction with a microRNA [60]. Several recent studies have supported the role of FMRP in modulating stability of its targets through $\mathrm{N}^{6}$-methyladenosine modification of the mRNA and codon optimality [54, 74]. The observation that DHPG results in decreased RPFs and TE on FMRP target mRNAs suggests they have reduced translation. This would be a surprising finding based on previous work that DHPG rapidly stimulates the synthesis of proteins encoded by FMRP target mRNAs such as Map1b, Arc, EF1a, and PSD-95 [22, 60, 65, 75]. Therefore, decreased RPF and TE may not always indicate reduced synthesis of protein. Some evidence indicates that FMRP stalls or slows ribosome translocation, and mGluR stimulation regulates translation elongation through FMRP and eEF2 phosphorylation $[18,59,76,77]$. Thus, for some mRNAs, the observed decrease in RPF or TE on FMRP target mRNAs in response to DHPG may be a consequence of enhanced ribosome movement that reflects increased protein synthesis.

Recent work has revealed regulation of translational elongation as critical for multiple forms of proteinsynthesis-dependent synaptic plasticity, including 
mGluR-LTD signaling [18, 78-80]. DHPG-induced mGluR1/5 signaling via ERK and AKT is normal in the $T s c 2^{+/-}$hippocampus, which mainly feeds into regulation of translation initiation [81]. Instead, signaling to ribosome translocation (polypeptide elongation) is altered in $T s c 2^{+/-}$and a low-dose cycloheximide enhances mGluR-LTD in these mice. Increased levels of phospho-eEF2 and low-dose cycloheximide have previously been shown to paradoxically increase translation of specific mRNAs, such as Arc and alpha-CAMKII, despite globally inhibiting protein synthesis $[18,80]$. Furthermore, we find evidence for a mechanism of occlusion via eEF2 phosphorylation in $T s c 2^{+/-}$, where some of the mRNAs and RPFs increased in $T s c 2^{+/-}$mice appear to no longer respond to DHPG, consistent with the higher basal levels of eEF2 and the deficient eEF2 response following DHPG. Our work suggests a novel mechanism of translational dysregulation in $T s c 2^{+/-}$and mGluR synaptic plasticity through signaling to ribosome elongation.

A combined $T s c 2^{+/-}$and $F m r 1^{-/ y}$ mouse rescues phenotypes in synaptic plasticity and contextual fear conditioning in each other, yet the mechanism of this rescue paradigm has remained elusive. In $T s c 2^{+/-}$and following DHPG-mGluR signaling, FMRP binding targets have decreased TE from increased RNA levels; in $\mathrm{Fmrl}^{-/ y}$, RNA levels of FMRP targets are instead decreased. Furthermore, DHPG-induced phospho-eEF2 is deficient in $T s c 2^{+/-}$, but enhanced in $F m r 1^{-/ y}$ mice [14]. This bidirectional alteration of FMRP binding targets and mGluR5 signaling to elongation suggest mechanisms for the opposing changes in synaptic plasticity and rescue paradigm of the $T s c 2^{+/-} / F m r 1^{-/ y}$ mice. Because total FMRP protein levels are unchanged in $T s c 2^{+/-}$, the dysregulation of FMRP binding targets may be through alternate pathways in transcription, mRNA stability, and/ or translation. Furthermore, mRNAs that are dysregulated in $T s c 2^{+/-}$are enriched for DHPG-mGluR-responsive mRNAs and many of these overlapping mRNAs are FMRP binding targets. These results reveal a complex interaction between TSC2 and FMRP in steady state and mGluR regulation of distinct mRNA subclasses.

\section{Limitations}

We anticipated that loss of one copy of Tsc2 would result in widespread but perhaps subtle gene expression changes, consistent with other mouse models of neuropsychiatric disorders. As such, we used a FDR $p$-adjusted cutoff of 0.1 for the genotype comparison. One limitation of the interpretation of our ribosome profiling results is that we identified altered signaling to ribosome movement in $T s c 2^{+/-}$and following mGluRLTD signaling. Thus, changes in ribosome density may not a priori reflect changes in protein synthesis. Multiple mouse models of autism have altered mGluR-LTD signaling, but evidence of altered mGluR-LTD in humans with autism has yet to be established. For Figs. 1 and 5, we utilized hippocampal slices prepared from 72 mice for a sample size of three per group, a standard practice in the ribosome profiling and RNA-seq field. Sequencing experiments with a sample size of 3 are limited by low power, and to reach a power of $>0.8$ would require a sample size of 30 libraries from over 600 littermate mice, which is prohibitive in cost for animals and reagents. The low power indicates that our datasets have a high probability of Type II errors (false negatives), and thus we would be unlikely to detect a genotype effect. This caveat, however, does not change the conclusions of our study. In any event, our findings need to be replicated in other studies.

\section{Conclusions}

By performing RNA-seq and ribosome profiling on a $T s c 2^{+/-}$mouse model and following induction of mGluRLTD, we identified changes in FMRP binding targets that suggest a molecular basis for bidirectional regulation of synaptic plasticity and behavior by TSC 2 and FMRP. Our study also suggests that altered mGluR-regulated translation elongation contributes to impaired synaptic plasticity in $T s c 2^{+/-}$mice.

\section{Supplementary information}

Supplementary information accompanies this paper at https://doi. org/10.1186/s13229-020-00384-9.

Additional file 1: Fig. S1. Validation of differential expression results from $\mathrm{TsC2}^{+/-}$versus wild type from Fig. 1. Histogram of the number of differentially expressed genes called from 4990 random permutations of sample labels for the genotype comparison from a RNA-seq and $\mathbf{b}$ ribosome profiling. Fig. S2. a Density plot of the length (left) and minimal free energy (MFE) (right) of the $5^{\prime}$ UTR of Down and Up RPFs in $\mathrm{TsC2}^{+/-}$compared to all mRNAs. ns nonsignificant, Wilcoxon rank-sum test with correction by Bonferroni method. $\mathbf{b}$ The ratios of phosphorylated/ total mTOR, rpS6, and 4EBP1 as determined by western blots of whole hippocampal lysates are unchanged in $\mathrm{TsC2}^{+/-}$mice as compared to wildtype littermates ( $n=13-15$ mice/genotype, $n$ s non-significant, unpaired t test). c Total levels of RPL4, RPS25, and LARP1 from hippocampus of wild-type and $T s c 2^{+/-}$littermates ( $n=13-15$ mice/genotype, $n s=$ nonsignificant, unpaired $t$ test). d Pearson correlation coefficient was computed for pairs of transcript features including translation efficiency (TE), coding length, and GC content (CDS), 5' untranslated region length and GC content ( $\left.5^{\prime} \cup T R\right), 3^{\prime}$ untranslated region length and GC content ( $3^{\prime} \cup T R$ ), and RNA abundance (TPM, transcripts per million). Red indicates a positive correlation, and blue a negative correlation. Gray indicates a correlation of identical parameters. $p<0.05$ for all correlation coefficients. Fig. S3. Gene set enrichment analysis of mGluR-responsive RNAs and translation. $\mathbf{a}, \mathbf{b}$ Gene set enrichment analysis of GO terms enriched (padj <0.05) in DHPG/ Basal RNAs (A) and RPFs (B). Gene lists were ranked by wild-type DHPG/ Basal expression changes (log2FC) and $p$ value. GO terms are separated by enrichment in either upregulated (increased) or downregulated (decreased) RNAs and RPFs. 
Additional file 2: Table S1. Table of GO terms enriched in $\mathrm{TsC}^{+/-} / \mathrm{WT}$ upregulated RNA and RPFs from Fig. 1D, E (padj < 0.05 for cellular compartment, molecular function, and biological process).

Additional file 3: Table S2. Table of GO terms enriched in wild-type DHPG/basal ranked list of RNA and RPFs expression changes from Supplemental Fig. 2 (padj < 0.05 for cellular compartment, molecular function, and biological process).

Additional file 4: Table S3. Table of DESeq2 output for RNA and RPF expression changes for TsC2 ${ }^{+/-}$WT and WT DHPG/Basal comparisons. FMRP binding targets are indicated in the last column based on binding strength (stringent, high, low). Nontargets are indicated by nonclip. Overlapping mRNAs and RPF altered in $\mathrm{TsC2}^{+/-}$and responsive to DHPG from Fig. 5 are listed.

Additional file 5: Table S4. Table of anota2seq output for RNA, RPF, and TE expression changes for TsC2 ${ }^{+/-} /$WT. The delta tab has calculated expression changes (log2FC) for RNA, RPF, and TE. The total mRNA tab has analysis for RNA expression changes (log2FC). The translated mRNA tab has analysis for RPF expression changes (log2FC). The buffering tab has analysis for TE expression changes (log2FC) that likely leads to translational buffering. The translation tab has analysis for TE expression changes ( $\log 2 \mathrm{FC})$ that likely leads to altered protein levels.

Additional file 6: Table S5. Table of anota2seq output for RNA, RPF, and TE expression changes for WT DHPG/Basal. The delta tab has calculated expression changes (log2FC) for RNA, RPF, and TE. The total mRNA tab has analysis for RNA expression changes (log2FC). The translated mRNA tab has analysis for RPF expression changes (log2FC). The buffering tab has analysis for TE expression changes (log2FC) that likely leads to translational buffering. The translation tab has analysis for TE expression changes ( $\log 2 \mathrm{FC})$ that likely leads to altered protein levels.

\section{Abbreviations}

CHX: Cycloheximide; DE: Differentially expressed; DHPG: Dihydroxyphenylglycine; eEF2: Eukaryotic elongation factor 2; FMRP: Fragile X Mental Retardation Protein; LTD: Long-term depression; mGluR: Metabotropic glutamate receptor; mTOR: Mechanistic target of rapamycin; RPF: Ribosome-protected fragment; TE: Translation efficiency; TOP: Terminal oligopyrimidine tract; TSC: Tuberous sclerosis complex; WT: Wild type.

\section{Acknowledgements}

We would like to thank members of the Richter Lab for helpful comments, Elisa Donnard for setting up the RiboSeq pipeline, Ruijia Wang, Julie Zhu, and Jeon Lee for help with the bioinformatics analysis, and Patricia Hahn, Courtney Saqueton, and Elaine Lai for technical assistance.

\section{Authors' contributions}

K.M.H. and J.D.R. conceived the study. G.M. collected and prepared the hippocampal slices, performed western blotting, and performed e-physiology experiments. A.H. and B.L. prepared the sequencing libraries. A.H. performed the sequencing data analysis, and G.M. performed the data analysis for the western blots and e-physiology experiments. A.H., J.D.R., G.M., and K.M.H. interpreted the results and wrote the manuscript with input from all authors. All authors read and approved the final manuscript.

\section{Funding}

This work was supported by a Simons Foundation Grant 400998 (to JDR and KH) and NIH Grants GM46779, HD082013 (to JDR).

\section{Availability of data and materials}

The datasets supporting the conclusions of this article are included within the article in Additional files 4, 5, 6. The RNA-seq and Ribo-seq datasets supporting the conclusions of this article are available in the GEO Database repository: GSE144539 and GSE158881. Publically available datasets analyzed in the current study are from the following GEO Database repository: Shah et al., 2020: GSE143333.

\section{Ethics approval and consent to participate}

All experiments were approved by the Institutional Animal Care and Use Committee at University of Texas Southwestern and conducted in accordance with the National Institutes of Health Principles of Laboratory Animal Care.

\section{Consent for publication}

Not applicable.

\section{Competing interests}

The authors declare that they have no competing interests.

\section{Author details}

1 Program in Molecular Medicine, University of Massachusetts Medical School, Worcester, MA 01605, USA. ${ }^{2}$ Medical Scientist Training Program, University of Massachusetts Medical School, Worcester, MA 01655, USA. ${ }^{3}$ Department of Neuroscience, University of Texas Southwestern Medical Center, Dallas, TX 75390, USA.

Received: 2 March 2020 Accepted: 23 September 2020

Published online: 14 October 2020

\section{References}

1. Lipton JO, Sahin M. The neurology of mTOR. Neuron. 2014;84(2):275-91.

2. Auerbach $B D$, Osterweil EK, Bear MF. Mutations causing syndromic autism define an axis of synaptic pathophysiology. Nature. 2011;480(7375):63-8.

3. Tavazoie SF, Alvarez VA, Ridenour DA, Kwiatkowski DJ, Sabatini BL. Regulation of neuronal morphology and function by the tumor suppressors Tsc1 and Tsc2. Nat Neurosci. 2005;8(12):1727-34.

4. Garami A, Zwartkruis FJ, Nobukuni T, Joaquin M, Roccio M, Stocker H, et al. Insulin activation of Rheb, a mediator of mTOR/S6K/4E-BP signaling, is inhibited by TSC1 and 2. Mol Cell. 2003;11(6):1457-66.

5. Zhang Y, Gao X, Saucedo LJ, Ru B, Edgar BA, Pan D. Rheb is a direct target of the tuberous sclerosis tumour suppressor proteins. Nat Cell Biol. 2003;5(6):578-81.

6. Saxton RA, Sabatini DM. mTOR signaling in growth, metabolism, and disease. Cell. 2017;168(6):960-76.

7. Shaw RJ, Cantley LC. Ras, PI(3)K and mTOR signalling controls tumour cell growth. Nature. 2006:441 (7092):424-30.

8. Brunn GJ, Hudson CC, Sekulic A, Williams JM, Hosoi H, Houghton PJ, et al. Phosphorylation of the translational repressor PHAS-I by the mammalian target of rapamycin. Science. 1997;277(5322):99-101.

9. Gingras AC, Kennedy SG, O'Leary MA, Sonenberg N, Hay N. 4E-BP1, a repressor of $\mathrm{mRNA}$ translation, is phosphorylated and inactivated by the Akt(PKB) signaling pathway. Genes Dev. 1998;12(4):502-13.

10. O'Roak BJ, Vives L, Girirajan S, Karakoc E, Krumm N, Coe BP, et al. Sporadic autism exomes reveal a highly interconnected protein network of de novo mutations. Nature. 2012;485(7397):246-50.

11. Iossifov I, O'Roak BJ, Sanders SJ, Ronemus M, Krumm N, Levy D, et al. The contribution of de novo coding mutations to autism spectrum disorder. Nature. 2014;515(7526):216-21.

12. Huber KM, Kayser MS, Bear MF. Role for rapid dendritic protein synthesis in hippocampal mGluR-dependent long-term depression. Science. 2000;288(5469):1254-7.

13. Hou L, Klann E. Activation of the phosphoinositide 3-kinase-Akt-mammalian target of rapamycin signaling pathway is required for metabotropic glutamate receptor-dependent long-term depression. J Neurosci. 2004;24(28):6352-61.

14. Ronesi JA, Collins KA, Hays SA, Tsai N-P, Guo W, Birnbaum SG, et al. Disrupted Homer scaffolds mediate abnormal mGluR5 function in a mouse model of fragile X syndrome. Nat Neurosci. 2012;15(3):431-40.

15. Huber KM, Gallagher SM, Warren ST, Bear MF. Altered synaptic plasticity in a mouse model of fragile X mental retardation. Proc Natl Acad Sci U S A. 2002;99(11):7746-50.

16. Chévere-Torres I, Kaphzan H, Bhattacharya A, Kang A, Maki JM, Gambello $\mathrm{MJ}$, et al. Metabotropic glutamate receptor-dependent long-term depression is impaired due to elevated ERK signaling in the $\triangle R G$ mouse model of tuberous sclerosis complex. Neurobiol Dis. 2012;45(3):1101-10. 
17. Bateup HS, Takasaki KT, Saulnier JL, Denefrio CL, Sabatini BL. Loss of Tsc1 in vivo impairs hippocampal mGluR-LTD and increases excitatory synaptic function. J Neurosci. 2011;31(24):8862-9.

18. Park S, Park JM, Kim S, Kim JA, Shepherd JD, Smith-Hicks CL, et al. Elongation factor 2 and fragile $X$ mental retardation protein control the dynamic translation of Arc/Arg3.1 essential for mGluR-LTD. Neuron. 2008;59(1):70-83.

19. Zhu PJ, Chen CJ, Mays J, Stoica L, Costa-Mattioli M. mTORC2, but not mTORC1, is required for hippocampal mGluR-LTD and associated behaviors. Nat Neurosci. 2018;21(6):799-802.

20. Sharma A, Hoeffer CA, Takayasu Y, Miyawaki T, McBride SM, Klann E, et al. Dysregulation of mTOR signaling in fragile $X$ syndrome. J Neurosci. 2010:30(2):694-702

21. Di Prisco GV, Huang W, Buffington SA, Hsu C-C, Bonnen PE, Placzek AN, et al. Translational control of mGluR-dependent long-term depression and object-place learning by elF2a. Nat Neurosci. 2014;17(8):1073-82.

22. Waung MW, Pfeiffer BE, Nosyreva ED, Ronesi JA, Huber KM. Rapid translation of Arc/Arg3.1 selectively mediates mGluR-dependent LTD through persistent increases in AMPAR endocytosis rate. Neuron. 2008;59(1):84-97.

23. Ingolia NT, Ghaemmaghami S, Newman JRS, Weissman JS. Genome-wide analysis in vivo of translation with nucleotide resolution using ribosome profiling. Science. 2009;324(5924):218-23.

24. Ehninger D, Han S, Shilyansky C, Zhou Y, Li W, Kwiatkowski DJ, et al. Reversal of learning deficits in a Tsc2+/- mouse model of tuberous sclerosis. Nat Med. 2008;14(8):843-8.

25. Sato A, Kasai S, Kobayashi T, Takamatsu Y, Hino O, Ikeda K, et al. Rapamycin reverses impaired social interaction in mouse models of tuberous sclerosis complex. Nat Commun. 2012;3:1292.

26. Hernandez O, Way S, McKenna J, Gambello MJ. Generation of a conditional disruption of theTsc2 gene. Genesis. 2007;45(2):101-6.

27. Jakkamsetti V, Tsai NP, Gross C, Molinaro G, Collins KA, Nicoletti F, et al. Experience-induced Arc/Arg3.1 primes CA1 pyramidal neurons for metabotropic glutamate receptor-dependent long-term synaptic depression. Neuron. 2013;80(1):72-9.

28. Heyer EE, Ozadam H, Ricci EP, Cenik C, Moore MJ. An optimized kit-free method for making strand-specific deep sequencing libraries from RNA fragments. Nucleic Acids Res. 2015;43(1):e2.

29. Yu G, Wang LG, Han Y, He QY. clusterProfiler: an R package for comparing biological themes among gene clusters. Omics. 2012;16(5):284-7.

30. Nakagawa S, Hauber ME. Great challenges with few subjects: statistical strategies for neuroscientists. Neurosci Biobehav Rev. 2011;35(3):462-73.

31. Bateup HS, Johnson CA, Denefrio CL, Saulnier JL, Kornacker K, Sabatini BL. Excitatory/inhibitory synaptic imbalance leads to hippocampal hyperexcitability in mouse models of tuberous sclerosis. Neuron. 2013;78(3):510-22.

32. Nelson SB, Valakh V. Excitatory/inhibitory balance and circuit homeostasis in autism spectrum disorders. Neuron. 2015;87(4):684-98

33. Basu T, Riordan KJO, Schoenike BA, Khan NN, Wallace EP, Rodriguez G, et al. Histone deacetylase inhibitors restore normal hippocampal synaptic plasticity and seizure threshold in a mouse model of tuberous sclerosis complex. Sci Rep. 2019;9(1):5266.

34. Antoine MW, Langberg T, Schnepel P, Feldman DE. Increased excitationinhibition ratio stabilizes synapse and dircuit excitability in four autism mouse models. Neuron. 2019;101(4):648-61.e4.

35. Potter WB, Basu T, O'Riordan KJ, Kirchner A, Rutecki P, Burger C, et al. Reduced juvenile long-term depression in tuberous sclerosis complex is mitigated in adults by compensatory recruitment of mGluR5 and Erk signaling. PLOS Biol. 2013;11(8):e1001627.

36. Morita M, Gravel S-P, Chénard V, Sikström K, Zheng L, Alain T, et al. mTORC 1 controls mitochondrial activity and biogenesis through 4E-BPdependent translational regulation. Cell Metab. 2013;18(5):698-711.

37. Ebrahimi-Fakhari D, Saffari A, Wahlster L, Di Nardo A, Turner D, Lewis TL Jr, et al. Impaired mitochondrial dynamics and mitophagy in neuronal models of tuberous sclerosis complex. Cell Rep. 2016;17(4):1053-70.

38. Peters JM, Struyven RR, Prohl AK, Vasung L, Stajduhar A, Taquet M, et al. White matter mean diffusivity correlates with myelination in tuberous sclerosis complex. Ann Clin Transl Neurol. 2019;6(7):1178-90.

39. Tsai P, Sahin M. Mechanisms of neurocognitive dysfunction and therapeutic considerations in tuberous sclerosis complex. Curr Opin Neurol. 2011:24(2):106-13.
40. Gandin V, Masvidal L, Hulea L, Gravel SP, Cargnello M, McLaughlan S, et al. nanoCAGE reveals $5^{\prime}$ UTR features that define specific modes of translation of functionally related MTOR-sensitive mRNAs. Genome Res. 2016;26(5):636-48.

41. Thoreen CC, Chantranupong L, Keys HR, Wang T, Gray NS, Sabatini DM. A unifying model for mTORC1-mediated regulation of mRNA translation. Nature. 2012:486(7396):109-13.

42. Svitkin YV, Pause A, Haghighat A, Pyronnet S, Witherell G, Belsham GJ, et al. The requirement for eukaryotic initiation factor $4 \mathrm{~A}$ (elF4A) in translation is in direct proportion to the degree of mRNA $5^{\prime}$ secondary structure. RNA. 2001;7(3):382-94.

43. Holz MK, Ballif BA, Gygi SP, Blenis J. mTOR and S6K1 mediate assembly of the translation preinitiation complex through dynamic protein interchange and ordered phosphorylation events. Cell. 2005;123(4):569-80.

44. Raught B, Peiretti F, Gingras AC, Livingstone M, Shahbazian D, Mayeur GL, et al. Phosphorylation of eucaryotic translation initiation factor 4B Ser422 is modulated by S6 kinases. EMBO J. 2004;23(8):1761-9.

45. Browne GJ, Proud CG. A novel mTOR-regulated phosphorylation site in elongation factor 2 kinase modulates the activity of the kinase and its binding to calmodulin. Mol Cell Biol. 2004;24(7):2986-97.

46. Redpath NT, Foulstone EJ, Proud CG. Regulation of translation elongation factor-2 by insulin via a rapamycin-sensitive signalling pathway. EMBO J. 1996;15(9):2291-7.

47. Gentilella A, Moron-Duran FD, Fuentes P, Zweig-Rocha G, Riano-Canalias F, Pelletier J, et al. Autogenous control of 5'TOP mRNA stability by $40 \mathrm{~S}$ ribosomes. Mol Cell. 2017;67(1):55-70.

48. Tcherkezian J, Cargnello M, Romeo Y, Huttlin EL, Lavoie G, Gygi SP, et al. Proteomic analysis of cap-dependent translation identifies LARP1 as a key regulator of 5'TOP mRNA translation. Genes Dev. 2014;28(4):357-71.

49. Mills EW, Green R. Ribosomopathies: there's strength in numbers. Science. 2017. https://doi.org/10.1126/science.aan2755.

50. Chan LY, Mugler CF, Heinrich S, Vallotton P, Weis K. Non-invasive measurement of mRNA decay reveals translation initiation as the major determinant of mRNA stability. Elife. 2018;7:e32536.

51. Sawicka K, Hale CR, Park CY, Fak JJ, Gresack JE, Van Driesche SJ, et al. FMRP has a cell-type-specific role in CA1 pyramidal neurons to regulate autismrelated transcripts and circadian memory. Elife. 2019;8:e46919.

52. Ceolin L, Bouquier N, Vitre-Boubaker J, Rialle S, Severac D, Valjent E, et al. Cell type-specific mRNA dysregulation in hippocampal CA1 pyramidal neurons of the Fragile $X$ syndrome mouse model. Front Mol Neurosci. 2017; 10:340.

53. van Driesche SJ, Sawicka K, Zhang C, Hung SKY, Park CY, Fak JJ, Yang C, Darnell RB, Darnell JC. FMRP binding to a ranked subset of long genes is revealed by coupled CLIP and TRAP in specific neuronal cell types. bioRxiv. 2019;762500.

54. Shu H, Donnard E, Liu B, Wang R, Richter JD. FMRP links optimal codons to mRNA stability in neurons. bioRxiv. 2020;801449.

55. Zalfa F, Eleuteri B, Dickson KS, Mercaldo V, De Rubeis S, di Penta A, et al. A new function for the fragile $X$ mental retardation protein in regulation of PSD-95 mRNA stability. Nat Neurosci. 2007;10(5):578-87.

56. Cho J, Yu N-K, Choi J-H, Sim S-E, Kang SJ, Kwak C, et al. Multiple repressive mechanisms in the hippocampus during memory formation. Science. 2015;350(6256):82-7.

57. Niere F, Wilkerson JR, Huber KM. Evidence for a fragile X mental retardation protein-mediated translational switch in metabotropic glutamate receptor-triggered Arc translation and long-term depression. J Neurosci. 2012;32(17):5924-36.

58. Huang J, Ikeuchi Y, Malumbres M, Bonni A. A Cdh1-APC/FMRP ubiquitin signaling link drives mGluR-dependent synaptic plasticity in the mammalian brain. Neuron. 2015;86(3):726-39.

59. Nalavadi VC, Muddashetty RS, Gross C, Bassell GJ. Dephosphorylationinduced ubiquitination and degradation of FMRP in dendrites: a role in immediate early mGluR-stimulated translation. J Neurosci. 2012;32(8):2582-7.

60. Muddashetty RS, Nalavadi VC, Gross C, Yao X, Xing L, Laur O, et al. Reversible inhibition of PSD-95 mRNA translation by miR-125a, FMRP phosphorylation, and mGluR signaling. Mol Cell. 2011;42(5):673-88.

61. Carlberg U, Nilsson A, Nygard O. Functional properties of phosphorylated elongation factor 2. Eur J Biochem. 1990;191(3):639-45. 
62. Acevedo JM, Hoermann B, Schlimbach T, Teleman AA. Changes in global translation elongation or initiation rates shape the proteome via the Kozak sequence. Sci Rep. 2018;8(1):4018.

63. Sossin WS, Costa-Mattioli M. Translational control in the brain in health and disease. Cold Spring Harb Perspect Biol. 2019;11(8):a032912.

64. Schneider-Poetsch T, Ju J, Eyler DE, Dang Y, Bhat S, Merrick WC, et al. Inhibition of eukaryotic translation elongation by cycloheximide and lactimidomycin. Nat Chem Biol. 2010;6(3):209-17.

65. Graber TE, Hebert-Seropian S, Khoutorsky A, David A, Yewdell JW, Lacaille $J$, et al. Reactivation of stalled polyribosomes in synaptic plasticity. Proc Natl Acad Sci U S A. 2013;110(40):16205-10.

66. Bilanges B, Argonza-Barrett R, Kolesnichenko M, Skinner C, Nair M, Chen $M$, et al. Tuberous sclerosis complex proteins 1 and 2 control serumdependent translation in a TOP-dependent and -independent manner. Mol Cell Biol. 2007;27(16):5746-64.

67. McManus CJ, May GE, Spealman P, Shteyman A. Ribosome profiling reveals post-transcriptional buffering of divergent gene expression in yeast. Genome Res. 2014;24(3):422-30.

68. Gismondi A, Caldarola S, Lisi G, Juli G, Chellini L, ladevaia V, et al. Ribosomal stress activates eEF2K-eEF2 pathway causing translation elongation inhibition and recruitment of terminal oligopyrimidine (TOP) mRNAs on polysomes. Nucleic Acids Res. 2014;42(20):12668-80.

69. Wang X, Li W, Williams M, Terada N, Alessi DR, Proud CG. Regulation of elongation factor 2 kinase by p90(RSK1) and p70 S6 kinase. EMBO J. 2001;20(16):4370-9.

70. Cheng Z, Mugler CF, Keskin A, Hodapp S, Chan LY-L, Weis K, et al. Small and large ribosomal subunit deficiencies lead to distinct gene expression signatures that reflect cellular growth rate. Mol Cell. 2019;73(1):36-47.

71. Khajuria RK, Munschauer M, Ulirsch JC, Fiorini C, Ludwig LS, McFarland SK et al. Ribosome levels selectively regulate translation and lineage commitment in human hematopoiesis. Cell. 2018;173(1):90-103.e19.

72. Richter JD, Coller J. Pausing on polyribosomes: make way for elongation in translational control. Cell. 2015;163(2):292-300.
73. Olde Loohuis NF, Ba W, Stoerchel PH, Kos A, Jager A, Schratt G, et al. MicroRNA-137 controls AMPA-receptor-mediated transmission and mGluR-dependent LTD. Cell Rep. 2015;11(12):1876-84.

74. Zhang F, Kang Y, Wang M, Li Y, Xu T, Yang W, et al. Fragile X mental retardation protein modulates the stability of its m6A-marked messenger RNA targets. Hum Mol Genet. 2018;27(22):3936-50.

75. Waung MW, Huber KM. Protein translation in synaptic plasticity: mGluRLTD. Fragile X Curr Opin Neurobiol. 2009;19(3):319-26.

76. Shah S, Molinaro G, Liu B, Wang R, Huber KM, Richter JD. FMRP control of ribosome translocation promotes chromatin modifications and alternative splicing of neuronal genes linked to autism. Cell Rep. 2020;30(13):4459-72.

77. Darnell JC, Van Driesche SJ, Zhang C, Hung KYS, Mele A, Fraser CE, et al. FMRP stalls ribosomal translocation on mRNAs linked to synaptic function and autism. Cell. 2011;146(2):247-61.

78. Verpelli C, Piccoli G, Zibetti C, Zanchi A, Gardoni F, Huang K, et al. Synaptic activity controls dendritic spine morphology by modulating eEF2dependent BDNF synthesis. J Neurosci. 2010;30(17):5830-42.

79. McCamphill PK, Farah CA, Anadolu MN, Hoque S, Sossin WS. Bidirectional regulation of eEF2 phosphorylation controls synaptic plasticity by decoding neuronal activity patterns. J Neurosci. 2015;35(10):4403-17.

80. Scheetz AJ, Nairn AC, Constantine-Paton M. NMDA receptor-mediated control of protein synthesis at developing synapses. Nat Neurosci. 2000;3(3):211-6.

81. Roux PP, Topisirovic I. Signaling pathways involved in the regulation of mRNA translation. Mol Cell Biol. 2018;38(12):e00070-118.

\section{Publisher's Note}

Springer Nature remains neutral with regard to jurisdictional claims in published maps and institutional affiliations.
Ready to submit your research? Choose BMC and benefit from:

- fast, convenient online submission

- thorough peer review by experienced researchers in your field

- rapid publication on acceptance

- support for research data, including large and complex data types

- gold Open Access which fosters wider collaboration and increased citations

- maximum visibility for your research: over $100 \mathrm{M}$ website views per year

At $\mathrm{BMC}$, research is always in progress.

Learn more biomedcentral.com/submissions 